Non-invasive classification of gas-liquid two-phase horizontal flow regimes using ultrasonic Doppler sensor and neural network

Baba Musa Abbagoni and Hoi Yeung

Oil and Gas Engineering Centre

School of Energy, Environment and Agrifood

Cranfield University, Cranfield, MK43 OAL,

Bedfordshire, United Kingdom 


\title{
Non-invasive classification of gas-liquid two-phase horizontal flow regimes using ultrasonic Doppler sensor and neural network
}

\author{
Baba Musa Abbagoni ${ }^{1}$ and Hoi Yeung ${ }^{1}$ \\ ${ }^{1}$ Oil and Gas Engineering Centre \\ School of Energy, Environment and Agrifood \\ Cranfield University \\ b.abbagoni@cranfield.ac.uk
}

\begin{abstract}
The identification of flow pattern is key issue in multiphase flow which encountered in the petrochemical industry. Gas-liquid two-phase flow is difficult to identify the gas-liquid flow regimes objectively. This paper presents a feasibility of a clamp-on instrument for objective flow regime classification of two-phase flow using an ultrasonic Doppler sensor and artificial neural network. It is on recording and processing of the ultrasonic signals reflected from the two-phase flow. Experimental data obtained on a horizontal test rig with total pipe length of $21 \mathrm{~m}$ long and $5.08 \mathrm{~cm}$ internal diameter carrying air-water two-phase flow under slug, elongated bubble, stratified-wavy and, stratified flow regimes. Multilayer Perceptron Neural Networks (MLPNNs) used for developing the classification model. The classifier requires features as input which is representative of the signals. Ultrasound signal features extracted by applying both power spectral density (PSD) and discrete wavelet transforms (DWT) methods to the flow signals. A classification scheme of "1-of- $C$ coding method for classification" was adopted to classify features extracted into one of four flow regime categories. To improve the performance of the flow regime classifier network, a second level neural network was incorporated by using output of a first level networks features as input features. Addition of the two network models provided a combined neural network models which has achieved higher accuracy than single neural network models. Classification accuracies evaluated in the form of both the PSD and DWT features. The success rates of the two models are: (1) using PSD features, the classifier missed three datasets out of 24 test datasets of the classification and scored $87.5 \%$ accuracy. (2) With the DWT features, the network misclassified only one data point and it was able to classify the flow patterns up to $95.8 \%$ accuracy. This approach has demonstrated success of a clamp-on ultrasound sensor for flow regime classification and it would be possible in industry practice. It is considerably more promising than other techniques as it uses of non-invasive and nonradioactive sensor.
\end{abstract}

Keywords

Two-phase Flow Regimes, Ultrasonic Doppler Sensor, Discrete Wavelet Transforms, Power Spectral Density, Neural Networks, Feature Extraction and Flow Regimes Classification 


\section{Introduction}

Multiphase flows ocurs found in many industrial processes such as petroleum production, power generation, thermal engineering and nuclear reactors. The characteristic used to describe single phase flow such as turbulence; velocity profile and boundary layer are not suitable for describing nature of multiphase flows (Corneliussen et al. 2005). Multiphase flows are cateogrised into flow regimes. These flows occur both in horizontal and vertical orientations. The flow regimes are developed based on the flow-line geometry and orientation, individual phase flow rates, and component transport properties (density, viscosity and surface tension (Rajan et al. 1993). Identification of the flow regimes in multiphase flow is essential to both efficient operation of the multiphase flow systems and determination of phase fractions (Arvoh et al. 2012). To group flow regimes according to their topological similarities, several mechanisms of the flow regimes classifiers or flow regimes descriptors havee been developed over the years. Typical flow regimes in the horizontal pipe flow are: slug, stratified, wavy, elongated bubble and annular flow patterns and flow vertical gas-liquid flow are: the bubbly, slug, churn and annular flow (Falcone et al. 2009). The process of the objective flow regimes identification from the sensor signals of the flow requires use of a pattern recognition technique.

The application of pressures fluctuations of the two-phase flow signals and statistical analyses for objective characterization was pioneered by Drahoš \& Čermák (1989). The two-phase flow signals from several pressures transducers have been analyzed for features extraction using Power Spectral Density for generating input variable for the neural network (Kv \& Roy 2012; Xie et al. 2004; Sun \& Zhang 2008). Other sensor signals have been used for flow regime classification using the statistical moment of the analysis. Such as conductance probe,(Hernández et al. 2006), radioactive images (Sunde et al. 2005). It has been found that the pattern recognition of flow regimes using pressure signals is fast enough to be used for online flow regime identification (Xie et al. 2004; Kv \& Roy 2012). However, these transducers are invasive sensors. Hence there is need for a non-invasive method of flow regimes classification for two-phase flow such as ultrasound or gamma. In addition, the review of methods of objective flow regime classification showed that the early methods used mechanistic models or empirical models. The flow patterns of the multiphase flow were identified using equation governing the physics of the fluid developed from the mechanistic models derived from the physics of the fluid. The process of identifying flow patterns using these model have disadvantage each flow regime has to be examined independently(Ozbayoglu \& Ozbayoglu 2009)

Ultrasonic technique based on the principle of pulse-echo intensity is widely used in gas/liquid interface detection and often, location of the interface is determined by the 
measuring the time of flight of the reflected wave (Chang \& Morala 1990). Wada et al. (2006) presented an ultrasonic method of two-phase flow pattern recognition based on the measurement of the instantaneous echo intensity profile along the ultrasonic beam. They comment that echo intensity of the flow measured by the integral of the difference in energy of single phase flow and the two-phase flow over the pipe diameter. The flow patterns from single phase flow to annular flow identified by the statistical distribution of the echo intensity. Murai et al. (2010) developed a pulse-echo ultrasonic technique to determine instantaneous liquid-gas interface detection.

Chakraborty et al. (2009) introduced a new ultrasonic method for measuring void fraction of two-phase flow using an ultrasonic sensor and twin signal processing methods based on time series analysis technique: symbolic dynamic filtering and analytical signal space partitioning for void fraction measurements and identification of flow regimes. An algorithm was built on the method of symbolic dynamic filtering to analyze the ultrasonic pulse echoes reflected off the bubbles. The pulse-echo technique of flow pattern identification is not complete a flow regime classification technique but an identification of the flow pattern (Jha et al. (2013). However, the authors comment that more research work on the computational and experimental work is required before the method can be deploying for industrial use. Another issue is that the ultrasound method applied is not intrusive though but it is invasive set up. Jha et al. (2013) extended the work of Chakraborty et al. (2009) and propose that the concept of ultrasonic pulse echo to be implemented in a clamp-on set up together with the symbolic dynamic filtering for industrial application.

Despite the feasibilities of using the pulse-echo ultrasound for the flow regime identification, the flow regimes identification is based on computational models. The computational methods for flow regimes identification employed sets of non-linear equations but often the equations are simplified. For practical applications, the simplified equations are not often used because it requires prior knowledge of several flow properties such as pipe diameter and pipe thickness which degrades in the cause of time (Meribout et al. 2010). The method of the pulse-echo ultrasound which is limited in liquid flow velocity information due to the restriction on the maximum measurable velocity using pule wave ultrasound by the Nyquist criterion (Evans \& McDicken 2000).

Ultrasound Doppler flow sensors which uses continuous wave of ultrasound signals also has a great potential of achieving non-invasive flow velocity measurement. The techniques of using continuous wave ultrasound have been existed in the medical ultrasound system. The techniques uses frequency shift representing the flow velocities to develop methods to predict multiphase flow regimes (Übeyli \& Güler 2005). In multiphase flow measurement, Kouame et al. (2003) present an application of continuous wave Doppler Ultrasound (CWDU) velocity measurement to two phase flow in pipes. They proposed use of the 
frequency resolution techniques to overcome the hindrance to the velocity profile measurement by the presence of coloured noise which introduces a significant obstacle to the classical frequency estimators. Pulse echo ultrasound techniques for two-phase flow measurement have limited liquid velocity information due to the restriction on the maximum measurable velocity using pule wave ultrasound by the Nyquist criterion (Evans \& McDicken 2000). Also, characteristics of the reflected wave are being influenced by the shape and size of the interface about the ultrasound wave length (Murai et al., 2010).

Artificial Neural Networks (ANNs) are often preferred over statistical methods of pattern recognition because of its fast responses and simplification (Mi et al. 2001). Also, the ANNs have good performance on pattern recognition due to their efficiency and available learning algorithms (Jain et al. 2000). Also, with regards to flow regime classification, ANN has advantages over other analytical tools such as Expert System and Clustering. The Expert Systems require prior information on the flow regime which could be affecting its objectivity. Similarly, the Clustering may not affect performance accurately due to its poor handling of transitional data points( $\mathrm{Hu}$ et al. 2011). Usually, the process of the neural network development is by training the network to recognise the measurement error in training data and then the netowrk tests on another set data. If the trained network is accurate enough then it is implemented for online measurement for prediction error correction (Liu et al. 2001). More importantly, neural network would offer a non-linear mapping between the ultrasound input signals and the predicted flow regimes. So the use of the ANN avoids the need for calibration of the multiphase flowmeter (Figueiredo et al. 2016).

Filletti \& Seleghim (2010) developed a numerical simulation measurement of interfacial area and volumetric fraction in two-phase flow using acoustic signal and artificial neural network to investigate the feasibility of application of the ultrasound system for clamp on flow measuring system. They found that the trained ANN models were able to estimate the values of the volumetric fraction and the interfacial area. Similarly, Figueiredo et al. (2016) employed an ultrasonic methodology based on pulse wave ultrasound transducers which is operates on the principle of signal attenuation detection. The ultrasound signal attenuation was analysed and incorporated with artificial neural network for flow pattern detection and void fraction measurement. They suggest that the flow regimes identification in the 2 -in pipe was limited to bubbly flow and slug flow only. The technology presented is appropriate for the detection of the gas volume fraction (GVF) and flow regimes determination in multiphase flow. However, there does not appear to be any consideration of the flow regimes excpet of the bubbly flow and slug flow. However, according to the authors' knowledge, however, studies on the two-phase flow regimes classification using a clamp-on continuous wave Doppler ultrasound have not been reported in the open literature. 
The main aim of this research was to investigate the feasibility of a non-invasive method of flow regimes classification using ultrasonic Doppler sensor and neural network. A continuous wave ultrasound Doppler sensor employed in this study, has recently been implemented for investigating the velocity characteristics of slug body and film in a two-phase gas-liquid slug flow. The results showed velocity characteristics of the slug flow obtained is in good agreement with other experimental methods (Fan et al. 2014). The present approach is by recording and processing of ultrasonic Doppler signals on the flow and then features are extracted using both power spectral density and wavelet transforms methods. These features are the inputs for the ANN models which process it for the flow regime classification. Multilayer perceptron neural network (MLPNN) with three layers, namely inputs, varying hidden layers and four output neurons, were developed to mapping the flow regimes. Four numerical outputs are selected to represents the flow regimes are as follows: the elongated bubble, slug, stratified flow \& stratified wavy flow (Übeyli \& Güler 2005; Subasi 2005; Kandaswamy et al. 2004). Despite the ANNs have limitation that they cannot perform accurately outside the range of the training sets, a combination of several neurons of the ANN will able to 'learn' and memorised the data original variability so as to function as an objective flow regime classifier. Therefore, ANN can still fulfil appropriately the requirement for multiphase flow monitoring processes such as the flow regimes classification and prediction of the individual phase flow rates in multiphase, effectively (Rosa et al. 2010).

\section{Experimental Setup and Procedures}

\subsection{Two-Phase Flow Test Rig}

A horizontal air-water test rig for two-phase flow assessments at the Cranfield University's flow laboratory was used to conduct these experiments. A schematic diagram of the test facility is in Figure 2-1. The flow loop includes a closed loop PVC pipeline of 50-mm internal diameter with total pipe length of $21-\mathrm{m}$ long. an air compressor providedt the air flow and water is pumped into the loop from a storage tank of $2 \mathrm{~m}^{3}$ capacity using a $40 \mathrm{~m}^{3} / \mathrm{hr}$. water pump. The flow rates of air and water are controlled by regulating hand valves and measured using a turbine gas flow meter (QFG 25B/B/EP1, Quadrina) while water flow rate is metered with an electromagnetic (EM) flow meter (Altoflux K280/0, Altometer). The measurement section for the two-phase flow is made up of Perspex pipes that allow visualization of the flow regimes. The clamp-on ultrasound Doppler flow sensor was fixed on the bottom of the pipe. 


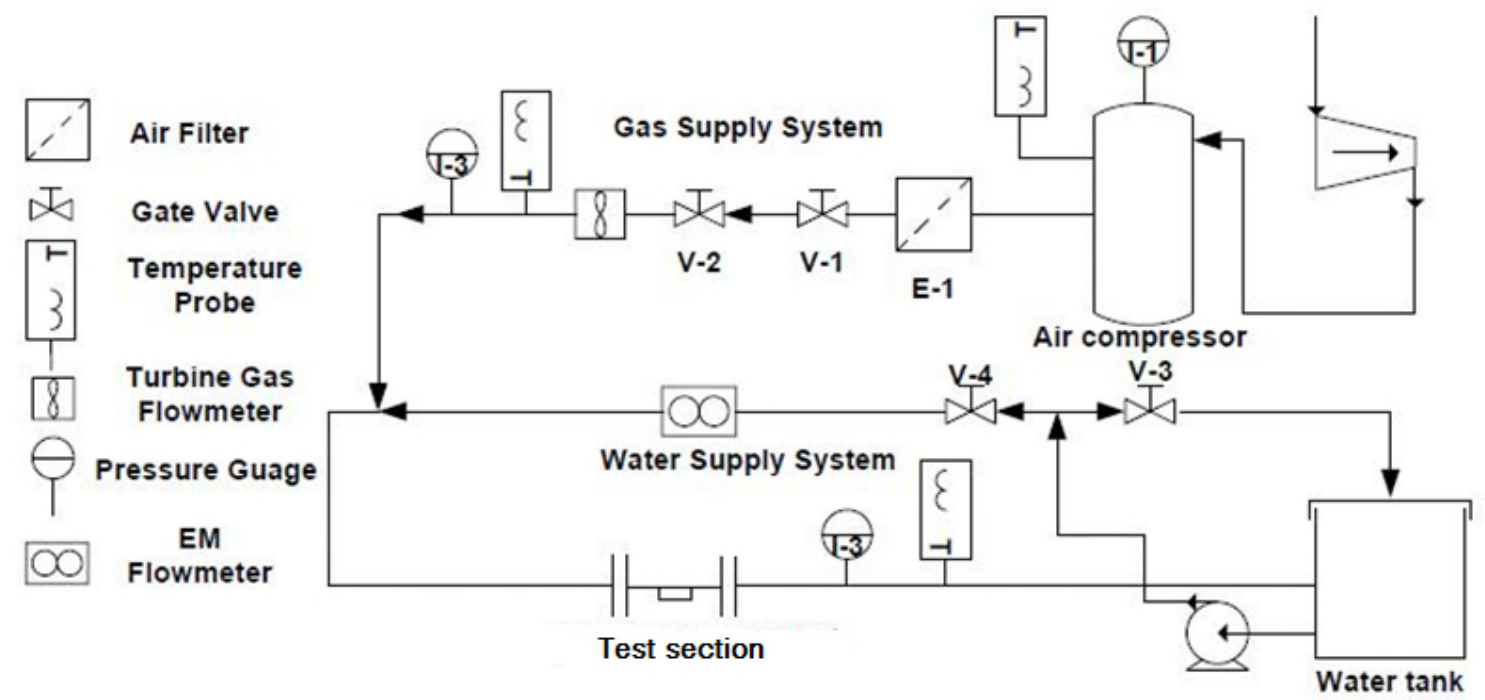

Figure 2-1 part of air-water test rig showing the instruments used in the experiment

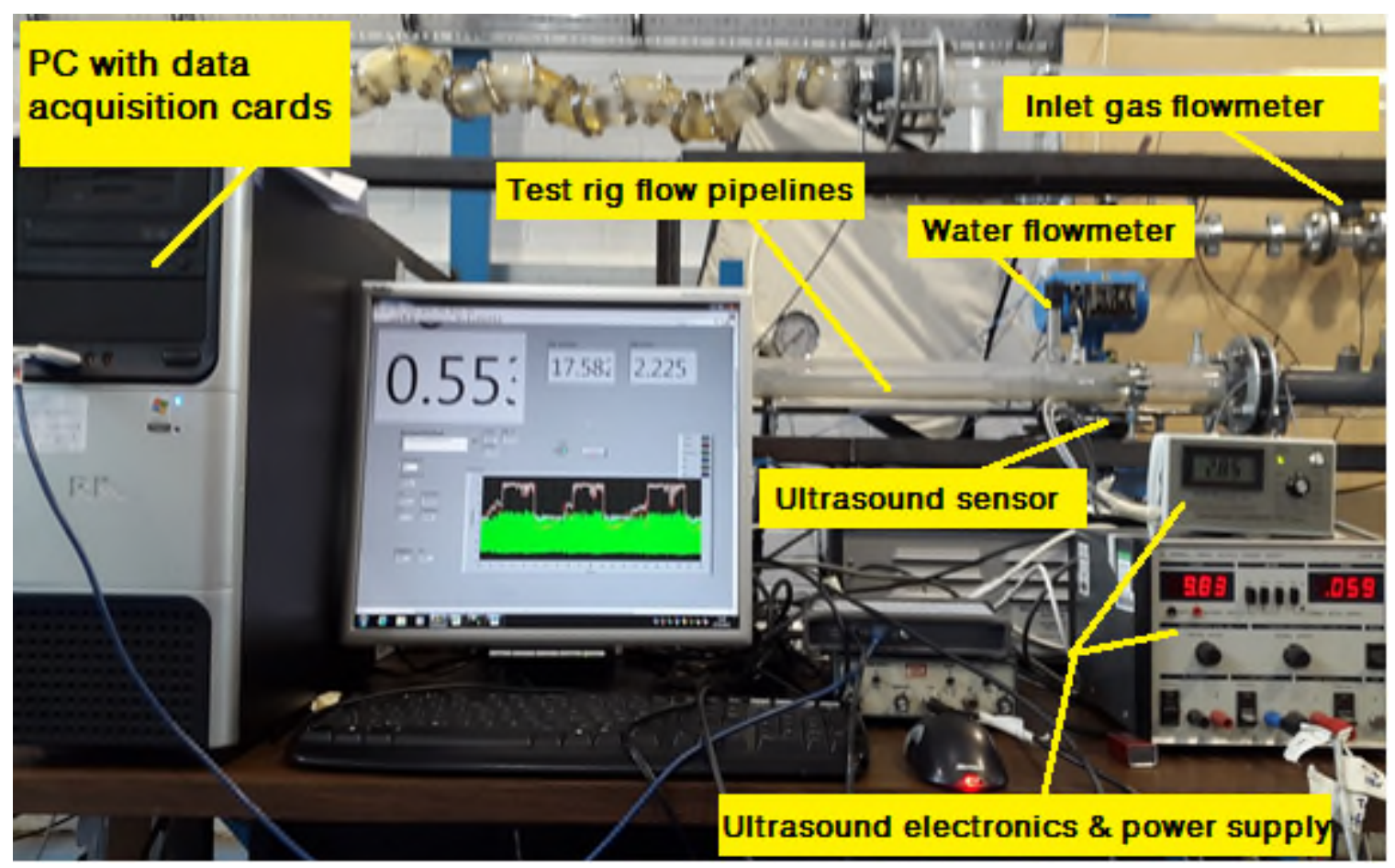

Figure 2-2 the ultrasonic Doppler sensor and its ancillary instruments on the flow test rig

\subsection{Ultrasonic Doppler senior}

The ultrasonic Doppler flowmeter used in this study is a non-invasive fluid flowmeter complete with its sensor, modelled as DFM-2 manufactured by United Automation Ltd, Southport, UK. This flowmeter is suitable for measuring the flow of any ultrasonic reflective fluid. It measures the frequency shift, process the signal, computes the flow velocity and gives out digital displays of the flow velocity in foot per seconds. A green LED shows the strength of the ultrasonic signal reflected back from the flow. It is recommended to be 
placed on the flow pipe at least ten diameters from bends, valves, tees, so as to prevent measurement errors from swirls, cavitation and turbulent eddies (UAL). The basic components of the Doppler flow meter system are shown in the Figure 2-4.The device measures the Doppler frequency shift of the ultrasonic signals reflected from the scatters or discontinuities such as bubbles in the flowing liquid. The sensor of the flowmeter placed at the bottom of the pipe at 6 o'clock position for the horizontal flow measurement test to avoid attenuation of the signal from gas voids in the upper pipe section. It is important to have good bonding between the sensor and the external pipe surface, a glycerine gel used for good coupling which prevents trapping of air cavities between the pipe surface and sensor. The continuous wave Doppler flow meter has two transducers: one for generating the sound wave and one for receiving the ultrasound reflected by the scatters in the fluids such as air bubbles or particles in the flow.

\subsection{Measurement principle}

The Doppler flowmeter systems used in this study has a transducer which has dual piezoelectric ceramic elements. The transducer is excited by the electronic circuit of the flowmeter in continuous mode, the transmitting part of the transducer sends out ultrasonic signal and the receiving part to detect the ultrasonic Doppler sensor provides the outputs signals. The output signal received is then filtered and amplified by the electronics of the flowmeter. The processed output signal is the Doppler frequency shift signal and it was captured using a data acquisition card (NI-PCl- 6040E) and LabVIEW program controlled sampling frequency of $10 \mathrm{kHz}$ for 20 seconds for each dataset. The process of development of the flow regimes classification described in a function blocks which various process involved in the system as shown in the Figure 2-3 


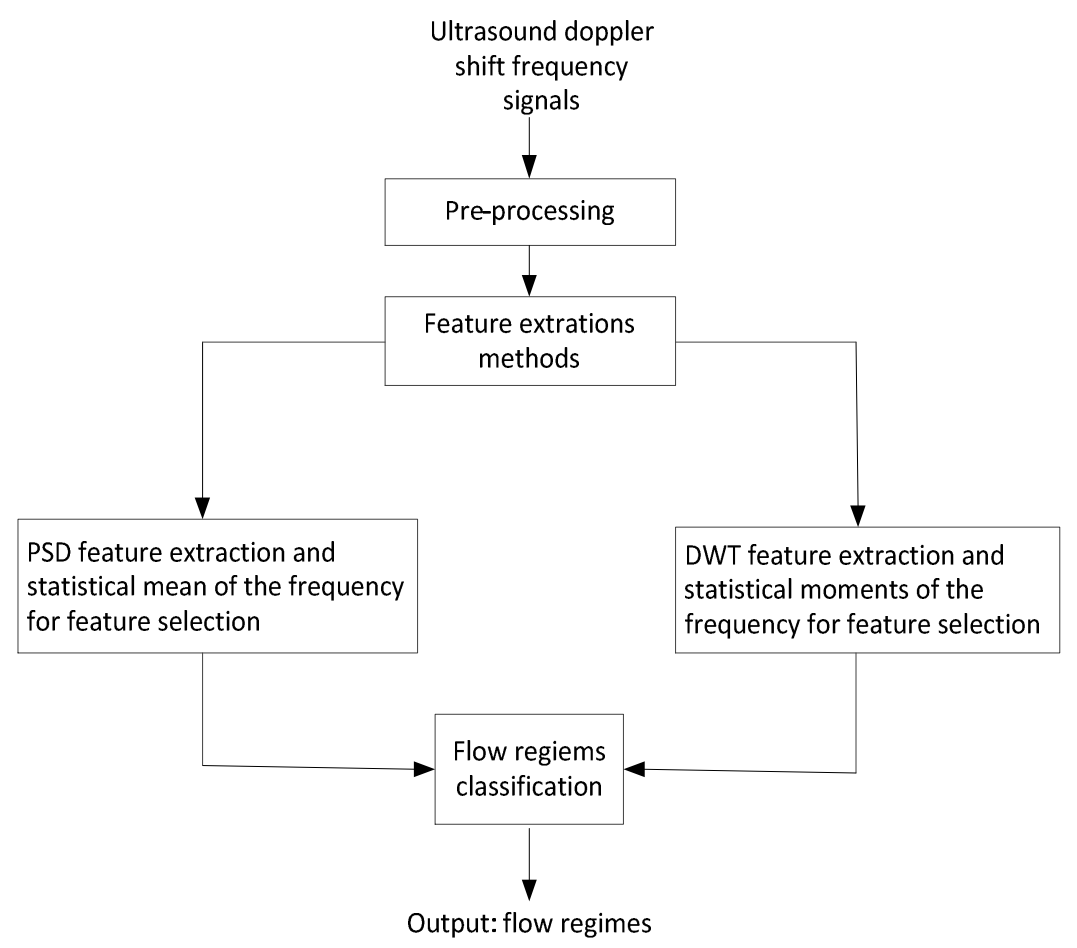

Figure 2-3 functional modules in the flow regime classification system

The relationship between the velocity of the scatters $v$ and the Doppler shift $f_{d}$ is given in equation (2-1)(Sanderson \& Yeung 2002).

$$
f_{d}=2 f_{t} \frac{v}{c} \cos \theta
$$

where: $v=$ average flow velocity, $c=$ velocity of sound in the fluid, $f_{d}=$ Doppler shift frequency, $\theta=$ angle between ultrasound beam and flow velocity and $f_{t}=$ ultrasound transmitted frequency.

Usually two transducers are required for Doppler flow meters. However, these two transducers can be made into separate units or one compact unit as this one.

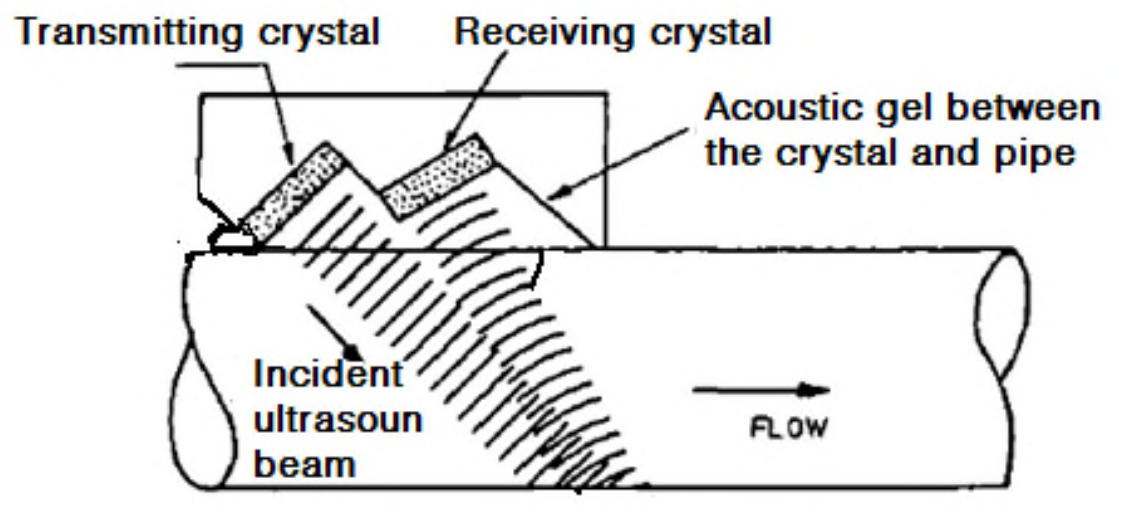

Figure 2-4 Schematic diagram of Doppler shift (Banerjee \& Lahey Jr 1981) 


\subsection{Ultrasonic flow signal data acquisition and Test Matrix}

Each of the test data set of the experiment created by setting the liquid flow rate to the desired value using a hand operated valve and the flow rate is measured using the EM flowmeter. First, the signal corresponding for this flow rate is recorded for calibration and air flow injected into the rig from an air compressor by regulating a valve by hand. The flow of the air supply is varied in steps. Thus generating several two phase sets in one particular liquid flowrate. The gas flow is measured with the turbine gas flowmeter. Temperature and pressure of the gas at the turbine meter location recorded for each flow. The superficial gas velocities ranged from $0.05 \mathrm{~m} / \mathrm{s}$ to $2.75 \mathrm{~m} / \mathrm{s}$.

All stated superficial gas velocities are at standard conditions ( 1 bar, average of $\left.22^{\circ} \mathrm{C}\right)$. The superficial velocity of the liquid flow was varied between $0.004 \mathrm{~m} / \mathrm{s}$ and $2.0 \mathrm{~m} / \mathrm{s}$. The superficial velocities values calculated as the ratio between the volumetric flow rate of the phase and the cross-sectional area of the pipe. These flow regimes characterized by distinct phase and velocity differences in the cross section of the pipe. Each of the two-phase flow of the dataset's flow regimes was visually observed, identified and recorded for comparison with predicted flow regimes. The total test data sets of the experiment tabulated as twophase test matrix includes the following flow regimes: Elongated bubble flow, slug flow, stratified flow and stratified wavy flow as shown in the Figure 2-5. The flow parameters recorded in the experiments are: the initial liquid flowrate, liquid superficial velocities, superficial gas velocities, ultrasonic reflected signals, temperature at the gas flowmeter, temperature at the test section, pressures at both gas and test section. The LabVIEW program was for controlling the data acquisition at sampling rate of $10 \mathrm{kHz}$ for $20 \mathrm{~s}$. The preliminary tests show that this sampling frequency is sufficient as it more than twice the highest frequency of the Doppler signals.

The preliminary tests show that this sampling frequency is sufficient as it more than twice the highest frequency of the Doppler signals.

A four-category classification chose for this purpose and the four regimes considered are:

- Stratified flow: when the liquid phase flow at the bottom and the gas phase is at the top, and interface of the two flow phases is smooth

- Stratified wavy flow: this flow occurs under condition whereby the gas velocity has risen to generate wave on the surface of the liquid.

- Slug flow: In the slug flow the liquid slugs are separated by the large gas bubbles moving violently downstream of the pipe.

- Elongated bubbly flow: this type of flow occurs when the flow has long gas bubble and short liquid slug(Chang \& Morala 1990; Canière et al. 2007): 


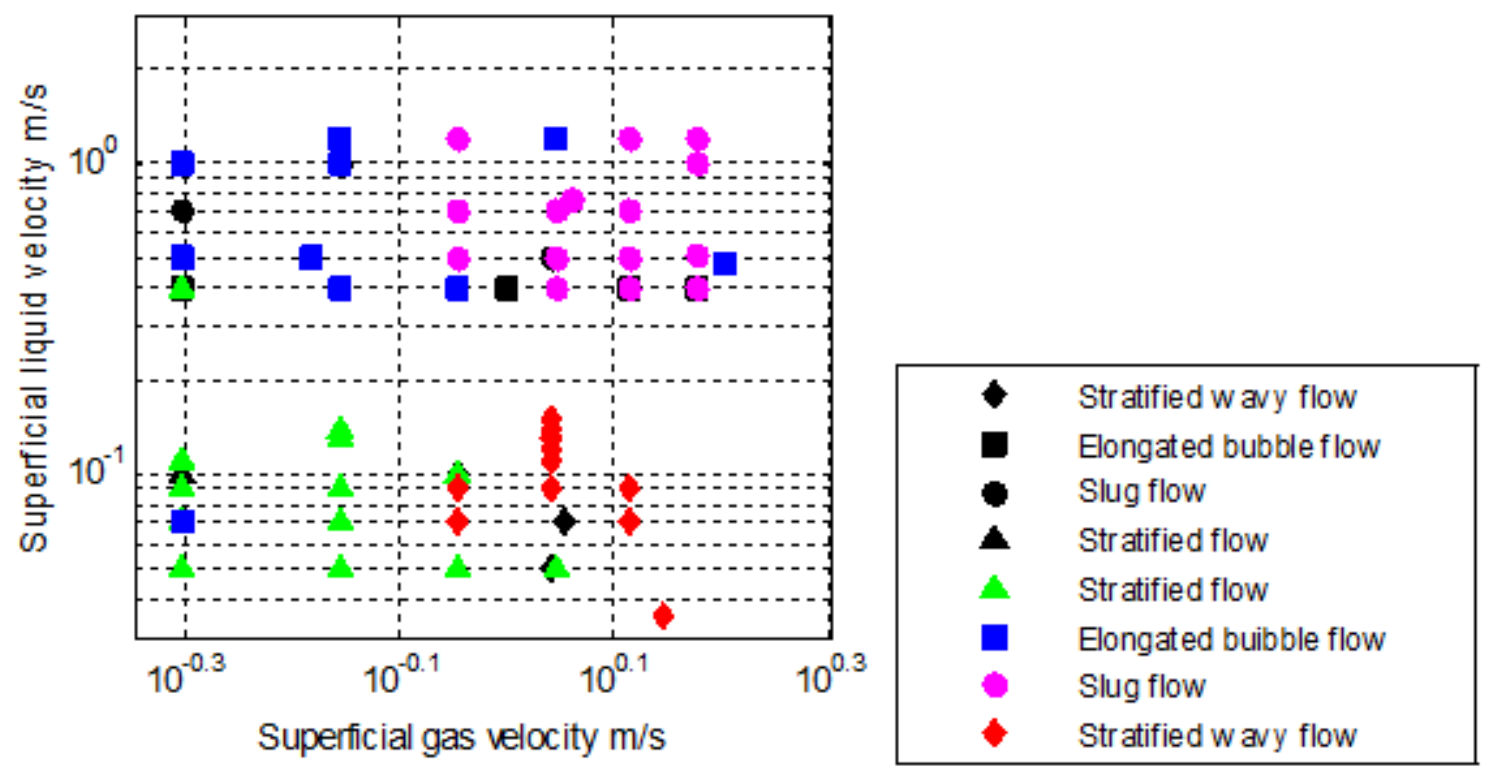

Figure 2-5 Flow regimes map of the present study (black shaded legend test data/ colour shaded legend training data)

\subsection{Spectral analyses and Feature Extraction}

Spectral analyses techniques are required for the analyses of the signals of two-phase flow to obtain the oscillation period. Two-phase flow signals can be analysed either in frequency domain, to obtain characteristics of the different flow regimes, algorithms such as power spectral density and wavelet transform which is time-frequency analysis used . In this work, both power spectral density and wavelet transform techniques have been applied to twophase flow signal records of the two-phase signals acquired using ultrasonic Doppler sensor (Shang et al. 2004). Frequency domain methods using the PSD have been used in analysing two-phase flow to get oscillation periods based on the Fourier transform of the signal (Xie et al. 2004). The wavelet transforms have the capability of analysing and denoising the signals to produce the spectrum in time-frequency domain.

\subsubsection{Power spectral density}

Frequency domain methods are often used to reveal the distinctiveness in the signal of flow regimes in two-phase flow systems. The power spectral density is a method of estimating characteristics of a time-series signal of stochastic process in the frequency domain that is suitable of detecting the frequency components hidden in the process (Matsumoto \& Suzuki 1984)(Matsumoto \& Suzuki 1984). The application of PSD to time series signal such as twophase flow pressure fluctuation signal has been studied by several researchers (Xie et al. 2004; Sun \& Zhang 2008; Santoso et al. 2012). The PSD is used to produce characteristics 
of the two-phase flow signal in the frequency domain which has shown that signals of the flow regime are distinctively different.

Fast Fourier transforms are use in creating the PSD spectrum which assumes that the process signal is stationary. The PSD function $P_{x}(f)$, of a discrete signal $x(n)$ is the Fourier transform of the autocorrelation sequence $R_{x}(k)$ of the signal as shown in the equation (Xie et al., 2004).

$$
P_{x}(f)=\sum_{-\infty}^{\propto} R_{x}(k) e^{-i 2 \pi f / f_{s}}
$$

The application of the PSD function to a real valued continuous data the autocorrelation sequence can be approximated by a time-average. However, in application of the function to measurement signal with is recorded for a finite time interval, this may present some distortions. As a consequence, a modified form of the PSD called Welch method is often adopted in these applications. The Welch method is by subdividing the signal sample into small length $\mathrm{N}$-points overlapping segment and then obtain the periodogram of each of the segments. The power spectrum is estimated by the average of the periodograms (Xie et al. 2004).

The Doppler ultrasonic frequency signals were processing in the in the MATLAB software package (MATLAB Version: 8.3.0.532) to analyse its spectral contents. Power spectral densities method using Welch method with segment length of 256 point and Hanning window to alleviate distortions is computing the spectra as applied by (Xie et al. 2004). Examples of the power spectra estimates of the samples of the signals shown in the Figure 2-6 and Figure 2-7. It can be seen that the spectrum of the slug flow signal has highest power on the spectrum. The slug flow ultrasound signal contains higher Doppler frequency shifts as well other the translational than the slug film velocity and the single phase flow. However, the spectrum of the signal of the single phase flow has produced a higher signal power than both stratified flow and bubbly flow signal but much less than that of slug flow signal. The rich ultrasonic shift frequency could be obtained from the signal phase flow because the flow pipe was full. Also, in the slug flow, there were intermittent full pipe and in the liquid slugs flow with higher velocity than corresponding single phase flow. The spectrum of the bubble flow regime is slightly similar pattern to that of slug flow. 

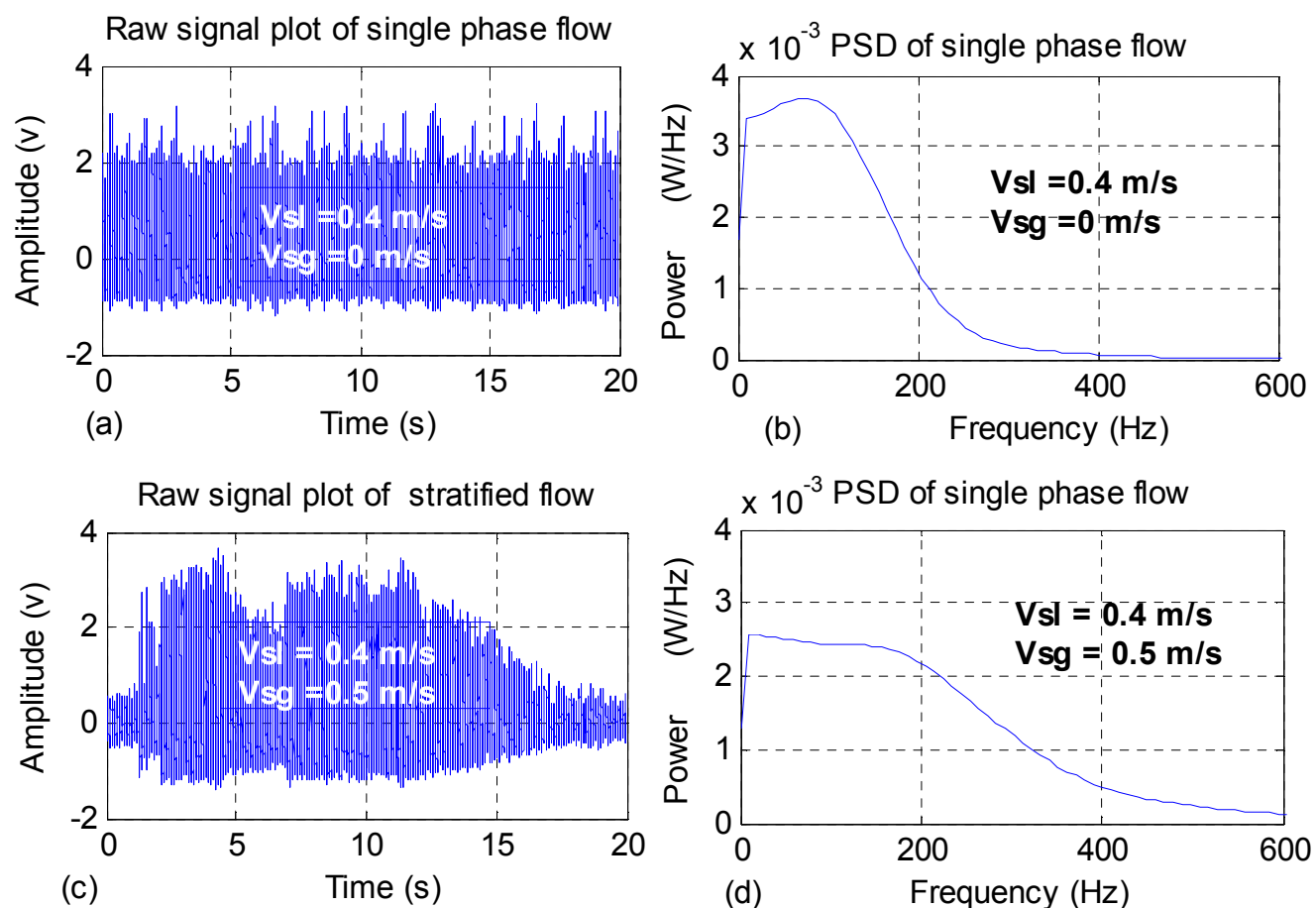

Figure 2-6 Single phase flow and stratified flow of an ultrasonic signal

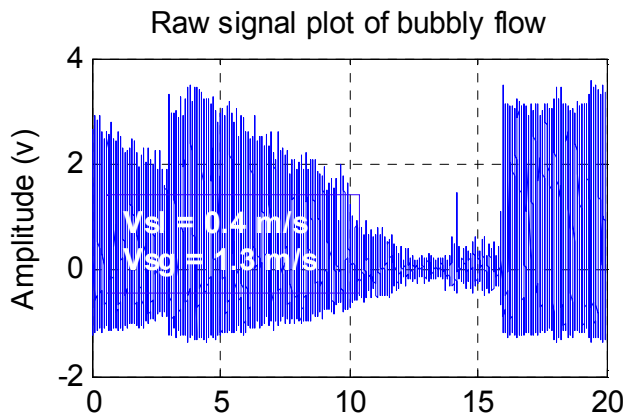

(a) Time (s)

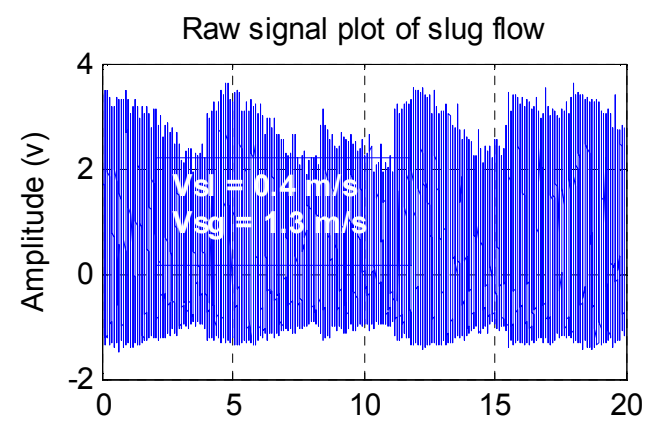

(c) Time (s)

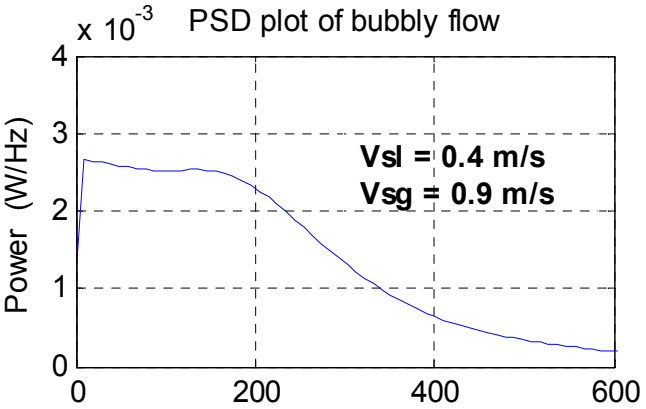

(b) Frequency $(\mathrm{Hz})$

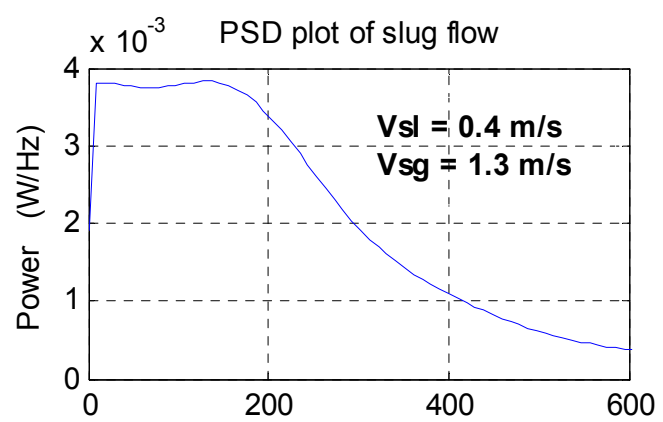

(d)

Frequency $(\mathrm{Hz})$

Figure 2-7 Bubbly flow and slug flow of an ultrasonic signal

The principle of extraction of the features from the PSD is based on Parseval's theorem which states that the PSD is the measure of the total energy of the signal if the spectrum is integrated over its entire frequency band. The sampling frequency and length of the signal 
play important role in the statistical properties. In the present study, the sampling frequency is chosen to be $10 \mathrm{kHz}$ so as to exceed the Nyquist criterion for the signal of the experiment. It can be seen in the Figure 2-7 The PSD spectrum produced from the signal recorded with ultrasonic Doppler sensor effective has frequency band from $0-600 \mathrm{~Hz}$. It is obvious that each of the PSD feature extracted are distinct and therefore, PSD feature is a good choice to be used to identify the flow regimes of the gas-liquid flow. As a result, the PSD magnitudes were normalised and their amplitudes corresponding to each data records in the frequency range. Each frequency bands of the two signals divided into five bands. PSD of Ultrasonic signal are partitioned into $120 \mathrm{~Hz}$ ranges as shown in Table 2-1

Table 2-1 Frequency Band

\begin{tabular}{llc}
\hline \multicolumn{3}{l}{ Ultrasonic signal PSD frequency band } \\
\hline Band name & Frequency range $(\mathrm{Hz})$ & Average power \\
\hline B1 & $0-120$ & $\bar{P}_{B 1}$ \\
B2 & $120-240$ & $\bar{P}_{B 2}$ \\
B3 & $240-360$ & $\bar{P}_{B 3}$ \\
B4 & $360-480$ & $\bar{P}_{B 4}$ \\
B5 & $480-600$ & $\bar{P}_{B 5}$
\end{tabular}

representative of each flow signals createed using a mean value the frequency bands of the PSD spectrum are computed as $\bar{P}_{B 1}, \bar{P}_{B 2}, \bar{P}_{B 3}, \bar{P}_{B 4}$, and $\bar{P}_{B 5}$ (Matsumoto \& Suzuki 1984). Other properties estimated from the spectrum are the weighted mean of the frequency over the entire band and the variance the mean frequency (Sun \& Zhang 2008). In total, the five power magnitudes, weighted mean frequency, and variance as used as the features to represent the signal of the flow. The mean spectral power equation (2-3) and

$$
\bar{f}=\frac{\sum_{i} f_{i} P_{x}\left(f_{i}\right)}{\sum_{i} P_{x}\left(f_{i}\right)}
$$

Variance of the spectral power equation (2-4)

$$
\sigma_{f}^{2}=\frac{\sum_{i}\left(f_{i}-\bar{f}\right)^{2} P_{x}\left(f_{i}\right)}{\sum_{i} P_{x}\left(f_{i}\right)}
$$


The use of these seven discrete parameters $\left(\bar{P}_{B 1}, \bar{P}_{B 2}, \bar{P}_{B 3}, \bar{P}_{B 4}, \bar{P}_{B 5}, \bar{f} \& \sigma_{f}^{2}\right)$ to represents the signal were first suggested Drahoš \& Čermák (1989) and the method has been implemented by many researchers such as Shaban \& Tavoularis (2014) and Xie et al. (2004).

\subsubsection{Discrete wavelet transform (DWT)}

The idea of signal decomposition using the DWT is not new. However, its usefulness lies in the ability to manipulate the wavelet coefficients to identify characteristics of the signal distinct from the original time signal (Subasi 2005). In this work, decomposition of the ultrasound Doppler signal and the conductance signal from the two-phase flow were carried out using the DWT. The procedure for extraction signal features using DWT to represent requires selection of the wavelet type and level, multiresolution decomposition and selection effective coefficients of the discrete wavelets of the decomposition to represent the signal(Shang et al. 2004).

Discrete wavelet transform of a signal for feature extraction is on the principle of multiresolution signal decomposition in which a signal is filtered using a half band high-pass filter and low-pass filters(Subasi 2005). There are number of different wavelets and their levels to choose for the decomposition of the signals. It is important to select the suitable wavelet type and level to structure the wavelet filter for the decomposition. Wavelet type selsection is by either visually inspecting the data for continuity or testing the various types of wavelets with signal and the most efficient one is selected. If it is discontinuous type then Harr or sharp or else a smooth wavelet such as Daubechies wavelets is recommend. Daubechies wavelets level2 was used to compute the wavelet coefficients of the signal in this study (Kandaswamy et al. 2004).

The decomposition of the signals produces approximations and details levels with different frequency bands by using a successive low-pass and high-pass filtering. These details level will not lose their information in the time domain (Bendjama et al. 2015). However, useful information can be obtained from the subbands of the dominant frequencies, so statistical measurements of the subbands are representatives of these details levels. The signals of the flow were decomposed continually until all the dominant frequency ranges viewed. The signals do not have any useful frequency below $40 \mathrm{~Hz}$ and that is why the decomposition ended at level 7 which is the level at frequency subbands greater than $40 \mathrm{~Hz}$. Therefore, the Doppler signal was decomposed into details coefficients of $D_{1}-D_{7}$ where 1-7 refers to the detail wavelet coefficient levels: first to seventh and the last approximation is $A_{7}$. The ranges of the frequencies subbands are given in the decomposition The Daubechies wavelet of the order 2(db2) was used to compute the wavelet coefficients of the signal. The computation of the DWT of the coefficient was done in MATLAB software package(Misiti et al. 1997) . 
Table 2-2 Ranges of frequency bands in the different wavelet decomposition levels

\begin{tabular}{clll}
\hline \multicolumn{2}{l}{ Ranges of frequency bands in wavelet decomposition } \\
\hline Decomposed signal & Number of samples & $\begin{array}{l}\text { Frequency } \\
(\mathrm{Hz})\end{array}$ & range \\
\hline$D_{1}$ & 100000 & $2500-$ & 5000 \\
$D_{2}$ & 50000 & $1250-$ & 2500 \\
$D_{3}$ & 25000 & $625-$ & 1250 \\
$D_{4}$ & 12500 & $312.5-625$ \\
$D_{5}$ & 6250 & $156.25-312.5$ \\
$D_{6}$ & 3125 & $78.125-156.25$ \\
$D_{7}$ & 1562.5 & $39.0625-78.125$ \\
$D_{8}$ & 781.25 & $19.53125-39.0625$ \\
\hline
\end{tabular}

For each of the datasets, details wavelet coefficients at the first level, second level and up to the seventh level were computed. Importantly, to reduce the size of feature extracted from coefficients, statistical measurements were applied to the values of $D_{1}, D_{2}, D_{3}$, $D_{4}, D_{5}, D_{6}$ and $D_{7}$ as implemented in the work of Übeyli \& Güler, (2005).

Table 2-3 the extracted features of four exemplary ultrasonic records from the four flow regimes

\begin{tabular}{|c|c|c|c|c|c|c|c|c|}
\hline \multirow[t]{2}{*}{ Dataset } & \multirow{2}{*}{$\begin{array}{l}\text { Extracted } \\
\text { features }\end{array}$} & \multicolumn{7}{|c|}{ Wavelet coefficients subbands (ultrasonic signals) } \\
\hline & & D1 & D2 & D3 & D4 & D5 & D6 & D7 \\
\hline \multirow[t]{3}{*}{ Stratified } & Maximum & 0.0069 & 0.0146 & 0.0328 & 0.0842 & 0.2348 & 0.6549 & 1.782 \\
\hline & Mean & $5.7 \mathrm{E}-06$ & $\begin{array}{l}3.4 \mathrm{E}- \\
05\end{array}$ & 8.9E-05 & $\begin{array}{l}2.5 \mathrm{E}- \\
04\end{array}$ & $\begin{array}{l}6.6 \mathrm{E}- \\
04\end{array}$ & 0.0019 & 0.0054 \\
\hline & Minimum & -0.0104 & $\overline{-}-0146$ & -0.0259 & -0.0684 & -0.1908 & -0.5353 & -1.4834 \\
\hline \multirow{3}{*}{ Bubble } & $\begin{array}{l}\text { Standard } \\
\text { deviation }\end{array}$ & 0.0017 & 0.002 & 0.003 & 0.0063 & 0.0173 & 0.0485 & 0.1365 \\
\hline & Maximum & 1.8351 & 2.7112 & 4.0595 & 6.4941 & 7.3507 & 7.5821 & 4.5808 \\
\hline & Mean & $1.2 \mathrm{E}-04$ & -0.001 & $-1.9 \mathrm{E}-04$ & -0.0064 & -0.0045 & -0.0024 & $4.8 \mathrm{E}-04$ \\
\hline \multirow{3}{*}{$\begin{array}{l}\text { Stratified } \\
\text { Wavy }\end{array}$} & Minimum & -1.8178 & $\overline{2} .9443$ & -4.2209 & -6.2225 & -7.8911 & -8.6795 & -5.8672 \\
\hline & $\begin{array}{l}\text { Standard } \\
\text { deviation }\end{array}$ & 0.1738 & 0.3981 & 0.8375 & 1.4791 & 1.4709 & 1.3086 & 0.9101 \\
\hline & Maximum & 0.0069 & 0.0146 & 0.0363 & 0.0891 & 0.2443 & 0.6854 & 1.9266 \\
\hline \multirow[t]{8}{*}{ Slug } & Mean & $2.6 \mathrm{E}-06$ & $\begin{array}{l}4.8 \mathrm{E}- \\
07\end{array}$ & $1.5 \mathrm{E}-06$ & $\begin{array}{l}1.5 \mathrm{E}- \\
06\end{array}$ & $\begin{array}{l}-3.8 \mathrm{E}- \\
05\end{array}$ & $-3.6 \mathrm{E}-05$ & $\begin{array}{l}-8.8 \mathrm{E}- \\
06\end{array}$ \\
\hline & Minimum & & - & & & & & \\
\hline & & -0.0069 & 0.0171 & -0.0432 & -0.1172 & -0.3384 & -0.9363 & -2.4544 \\
\hline & $\begin{array}{l}\text { Standard } \\
\text { deviation }\end{array}$ & 0.0017 & 0.002 & 0.0031 & 0.0064 & 0.0178 & 0.05 & 0.1403 \\
\hline & $\begin{array}{l}\text { Maximum } \\
\text { Mean }\end{array}$ & 2.2339 & 3.3618 & 4.7681 & 6.8243 & 8.841 & $\begin{array}{l}9.4888 \\
2.11 \mathrm{E}-\end{array}$ & 5.9295 \\
\hline & Minimum & 0.0011 & $\begin{array}{l}0.0021 \\
-\end{array}$ & 0.0028 & -0.016 & -0.0193 & 04 & 0.0036 \\
\hline & & -2.1424 & 3.3179 & -4.889 & -6.8878 & -8.6584 & -9.5898 & -6.7651 \\
\hline & $\begin{array}{l}\text { Standard } \\
\text { deviation }\end{array}$ & 0.4739 & 0.9882 & 1.9812 & 3.2405 & 3.4286 & 2.3328 & 1.7116 \\
\hline
\end{tabular}


1. Maximum of the wavelet coefficients in each subband.

2. Mean of the wavelet coefficients in each subband.

3. Minimum of the wavelet coefficients in each subband.

4. Standard deviation of the wavelet coefficients in each subband.

Features 1-3 represent the frequency distribution of the signal and the feature four the amount of changes in frequency distribution. These are the statistical features used to represent the two-phase flow and as inputs into the neural network for the flow monitoring (Übeyli and Güler, 2005). The wavelet detail coefficients of the signals are distinctly different. Figure 2-8 Show the details wavelet coefficients corresponding to the $D_{1}$ frequency of the two-phase flow.

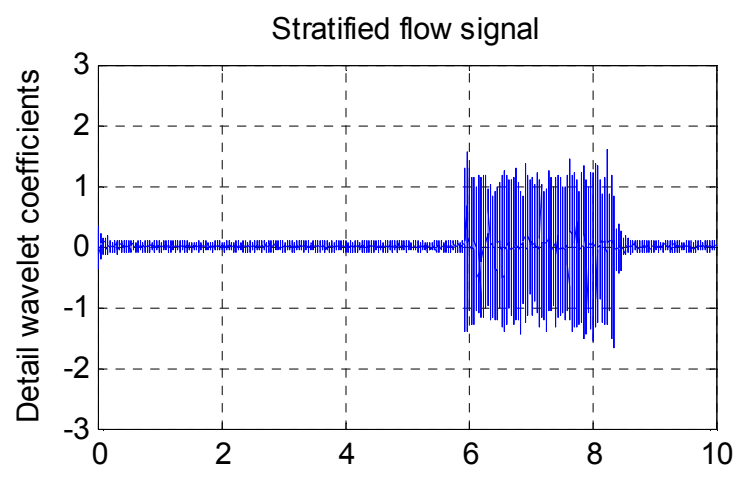

(a) Number of detail wavelet coefficients $\times 10^{4}$

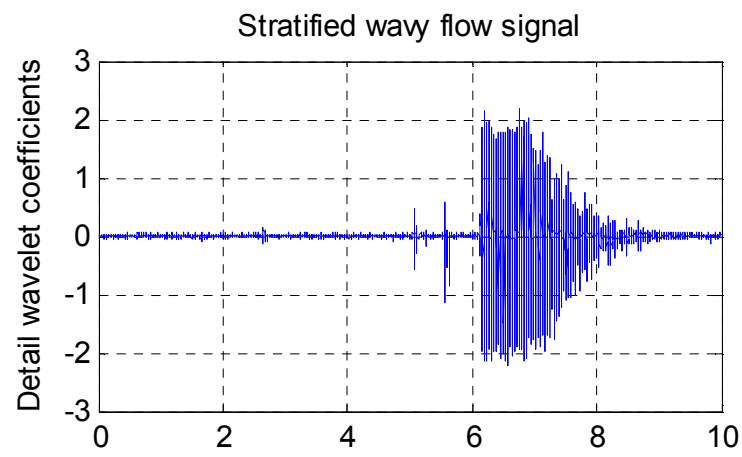

(c) Number of detail wavelet coefficients $\times 10^{4}$

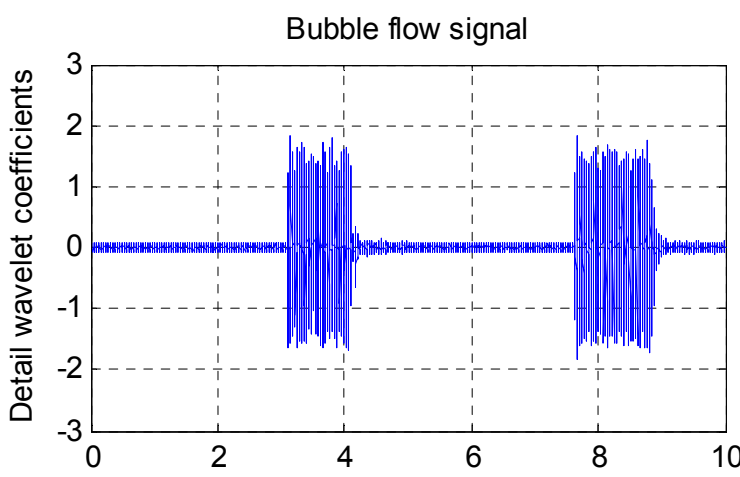

(b) Number of detail wavelet coefficients $\times 10^{4}$ Slug flow signal

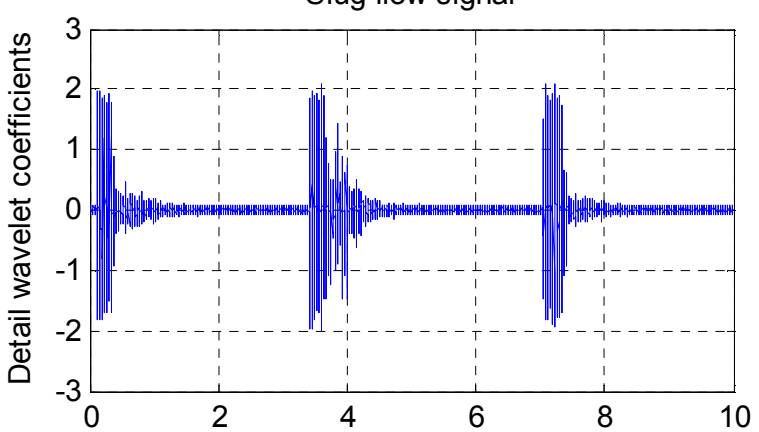

(d) Number of detail wavelet coefficients $\times 10^{4}$

Figure 2-8 the detail wavelet coefficients corresponding to the D1 frequency band of the ultrasonic signals from (a) stratified flow (b) Bubble flow (c) Stratified wavy flow and (d) Slug flow regime

\section{Multilayer perceptron neural network model (MLPNN)}

Multilayer perceptron neural network is a nonparametric technique for conducting various processing techniques for solving function approximation, pattern recognition, classification and estimations problems and its operation governed by a set of weights and biases (Übeyli and Güler, 2005). The general structure of the MLPNN with two successive layers is show in the Figure 3-1. The structure of the MLPNN model can be represented by the equation (3-1). 
The hidden layer is the unit in between the input layer and output layer. Its adjustments are not accessible from outside of the network(Luntta \& Halttunen 1999).

$$
y_{j}=f\left(\sum \mathrm{w}_{\mathrm{ji}} \mathrm{x}_{\mathrm{i}}\right)
$$

Where, $f$ is the activation function which transforms the weighted sums of all the input signals on the neurons. The activation function (f) can be taking many forms such as: threshold functions, or a sigmoidal, hyperbolic tangent or radial basis function. The sigmoidal function is one chosen for this study.

$$
f(\xi)=\frac{1}{1+e^{-\xi}}
$$

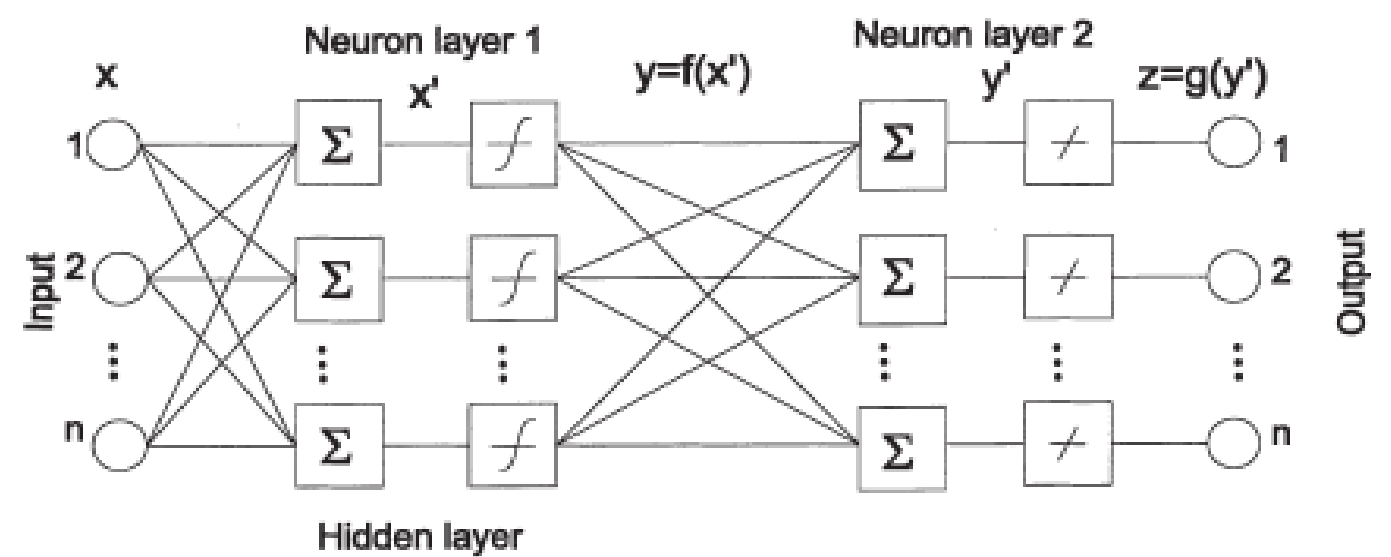

Figure 3-1 Multilayer perceptron neural network (Luntta \& Halttunen 1999)

The performance of an MLPNN can be improved by adjusting the weights of the network to reduce $E$ the difference between the desired output and the actual values of the neurons as fast as possible.

$$
E=\frac{1}{2} \sum_{j}\left(y_{d j}-y_{j}\right)^{2}
$$

Where, $y_{d j}$ is the desired value of the output neuron $j$ and is the actual output value whose values can be adjusted and then chosen using the set of targeted outputs.

The flow regimes classification with the ANN is implemented using pattern recognition algorithms. The pattern recognition comprises three steps: 1) data acquisition and preprocessing, 2) data representation or feature extraction, and 3) decision making or pattern classifying. One important aspects of the pattern recognition is the learning from the training data set(Basheer \& Hajmeer 2000). In this study, the training process consists of determination of the MLPNN model parameters which are used to validate their quality and ability to classify once the training has completed (Subasi, 2005). Alternatively, training 
refers to the process of adjusting and selecting the appropriate weights and biases(Bishop \& James 1993).

\subsection{Combined neural network models (CMLPNN)}

A combined neural network is a method of improving the performance of the network's predictive accuracy. In designing neural network models, the training data may fail to learn to predict the output accurately, so the network is unable to generalise the concept precisely. The learning system of the network utilises the transformed data to predict the output greater accuracy. Stack generalisation is a method of combining low level network models into high level neural network to achieve greater predictive accuracy introduced by Wolpert (1992). By transforming the data into a suitable form that can enhance the training process The generalisation minimizes error rate of the combined network by 'teaching' a second level network whose inputs are prediction of the first level network but the second trained with the same target output as the first network..

Multilayer perceptron neural network were used to form multiple networks and then combined to form a stack generalisation. Figure 3-2 shows the structure of the combined network. The features extracted from the sensors signals used as the input to the first level network of the combined neural network. After that the outputs of the first level were fed into second level network as the inputs. The outputs of the second levels are result of the predicted flow regimes. Both the first level and second level neural networks trained with targeted outputs (Übeyli and Güler, 2005).

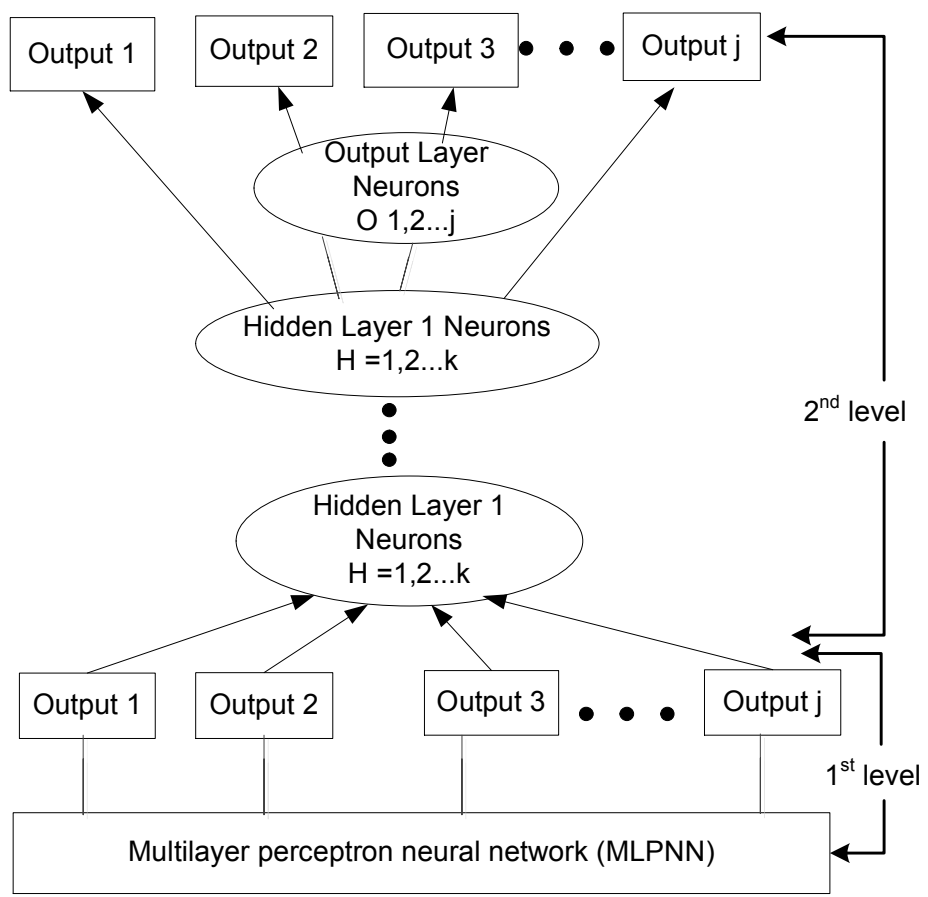

Figure 3-2 A second-level neural network is used for combine the predictions of the firstlevel neural networks(Übeyli \& Güler 2005) 
Multilayer perceptron neural network were used to form multiple networks and then combined to form a stack generalisation approach to the flow regimes classification. Figure 3-3 shows the structure of the combined network used predict targeted flow regimes. Both the first level and second level neural networks trained with targeted superficial flow velocities as the outputs.

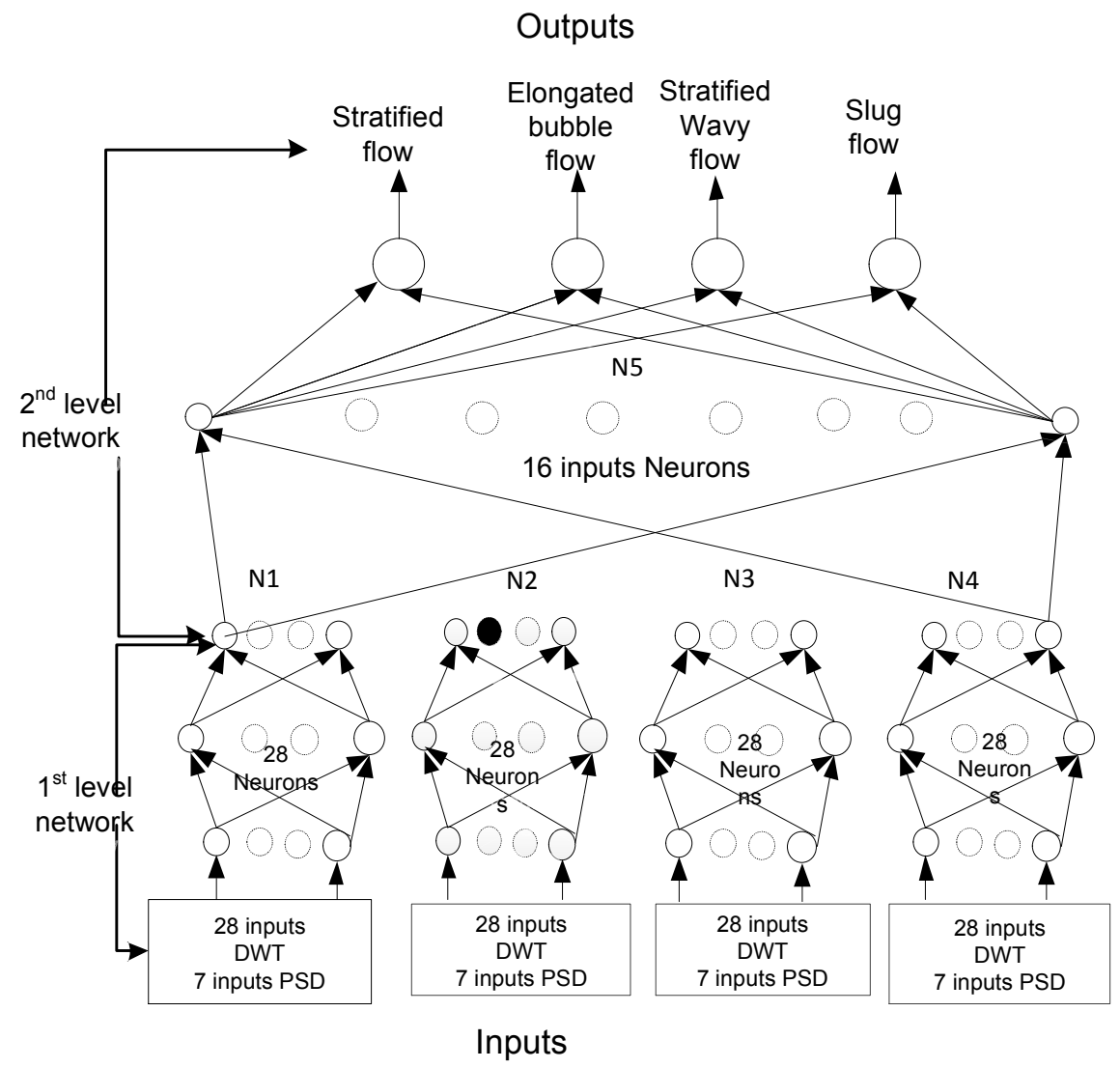

Figure 3-3 A Structure of the flow pattern prediction combined neural network topology (Übeyli and Güler, 2005).

\subsection{Flow Regime Classification network}

Several application of the MLPNN pattern recognition reported in the literature. It is based on the training the neural network to recognise the correct classification for each member of the training datasets. Training the network will be subsequently followed by testing the network to classify the input variable into their correct classes. If the learning process has taught the network the patterns relevant to the test data, then it is expected that the network would classify correctly (Xie et al., 2004).

Flow regime identification using ANN pattern recognition can be implemented by either using a supervised neural network(SNN) or unsupervised neural network(UNN) aslo known as self-organising network network. The SNN uses Feed-forward networks such as multilayer perceptron and Radiial-Basis Function networks for pattren recognition in which back propagation error of the training algorithm together information of predefined classes to 
classify the input variables into the specific classes and it does not need. Whereas Kohonen-Network or self-organizing map (SOM) used for data feature mapping and it does not need information of the classes. It uses network clustering method to group the input variable into several classes that contain similar characteritic(Mi et al. 2001). The Kohen Self-Organizing Neural Network has implemented for flowregime classification using measuremnt data pionts of disticntc flow regimes (Cai et al. 1994).

To select the input to the network for the pattern classification, it is essential first to preprocess, balance and normalise the data. The features extracted from the ultrasonic Doppler signal of the flow using both the wavelet method and the power spectral density methods are pre-processed before presenting them as input variables to the network. As part of the preprocessing the input dataset, data partitioning and balancing are applied to the feature. There several ratios for partitioning the datasets into training, testing and validation. At this moment, there is no mathematical rule for determining the exact sizes of the training, testing and validation datasets. The often used ratios are $60 \%, 30 \%$ and $10 \%$ or $65 \%, 25 \%$ and $15 \%$ for training, testing and validation respectively (Basheer \& Hajmeer 2000).

Another aspect of the preparing the input data is the balancing datasets which is distributing the training nearly evenly amongst the various classes to annul the effect of network from being biased to overrepresented classes. Input data preparation, first balancing and then normalisation process to prevent chaos in the network as a result of either the larger numbers overriding smaller ones or premature saturation of hidden nodes. Normalisation usually confines the data into uniform range 0 to 1 . A good rule of the thumb is to scale the input variables $\left(z_{i}\right)$ and the output range $\left(\lambda_{2}, \lambda_{1}\right)$ in interval of the output values which corresponding to the function in equation (3-3) (Basheer \& Hajmeer 2000).

$$
x_{i}=\lambda_{1}+\left(\lambda_{2}-\lambda_{1}\right)\left(\frac{z_{i}-z_{i}^{\text {min }}}{z_{i}^{\text {max }}-z_{i}^{\text {min }}}\right)
$$

Statistical analysis of the features plays important role in selecting the input variables for successful neural network application. Güler \& Übeyli (2006) computed the statistical features (mean, maximum, minimum and standard deviation) to represent the timefrequency features extracted from the Doppler signals using the wavelet transform.

The output of the neural network is the indicator of the flow regimes or classes of the classification which is represented with a continuous or binary discrete number. Each of the input variables to the neural network is assigned to the class to which it belongs. Usually, these classes are represented with numerical values. There are two most common representations of the classes: continuous $(0.3,0.5,0.7$, etc.) or discrete ( 0 and 1 or 0.1 and 0.9). Xie et al. (2004) have implemented the continuous number to represent the output of the neural network to indicate flow regimes. They used continuous numbers $(0.3,0.5,0.7$ 
and 0.9) to represent the flow regimes: bubbly flow, plug flow, churn-intermittent and slug flow respectively. This techniques was supported by their earlier work(Xie et al. 2003). The continuous designation number to represent the output of neural network flow classification methods of has been applied by other researchers as well (Sun \& Zhang 2008).

However, due to importance of the discrete output for extracting rules from trained neural network, Basheer \& Hajmeer (2000) have suggested that the continuous variable to be replaced by discrete or binary numbers for representing output of neural network classifier. There are methods and algorithms for discretizing the output variables. The discrete or binary number is often to modified from the $(0$ and 1$)$ to $(0.1$ and 0.9$)$ so as to prevent saturation(Basheer \& Hajmeer (2000). Also by allocating the targets of 0.1 and 0.9 instead of the common practice of 0 and 1 prevents the outputs of the network from directly interpretable posterior (Kandaswamy et al., 2004).

Most frequently used training model in classification problems in the back propagation (BP) which is adopted for this investigation and in other works (Fan \& Yan 2014; Blaney \& Yeung 2008;Arubi 2011). The MLPNN has properties such as the abilities to learn and transform fewer training set requirements and fast processing. The manner in which the weights can be adjusted governed by different training algorithms (Übeyli and Güler, 2005). The training tool has functions for the performance, the magnitude of the gradient of performance and the number of validation checks. The magnitude of the gradient and the number of validation checks are used to terminate the training. The gradient will become very small as the training reaches a minimum of the performance. If the magnitude of the gradient is less than 1e-5, the training will stop(Beale et al. 2013).

Cross validation in neural network pattern recognition are required to determine the optimum number of hidden units and the model that will perform best on the problem at hand (Bishop \& James 1993; Kandaswamy et al. 2004). A best network model for the flow regime classification obtained after testing several training algorithms. The performance test of the input datasets is determined by the computation of the total classification accuracy and number of training epochs. Total classification accuracy is number of correctly classified flow regimes/number of total datasets (Übeyli and Güler, 2005).

\subsection{Flow regimes classifier neural network training and testing}

Eighty six datasets measurements on the horizontal two-phase flow used for this experiment. Sixty-two datasets used for training the networks and twenty four are used for the testing the network. Two sets of combined neural network models developed in MATLAB software package (MATLAB Version: 8.3.0.532 (R2014a) with neural network toolbox) for classifying the flow regimes of air-water two-phase flow using features from ultrasonic signals of the flow. The inputs to the network are features extracted from the signals of the 
flow using both power spectral density and discrete wavelet transforms. The outputs of the combined neural network are (discrete binary) indicators of the four flow regimes: elongated bubble flow, slug flow, stratified flow and stratified wavy flow.

In this training process, classification scheme of "1-of-C coding method for classification" was adopted to classify the inputs and each of members of the datasets belongs to one output of the four flow regime categories. The four predetermined output values are as designated in the following equations: (3-5), (3-6), (3-7) and (3-8). The values are the targets presented to the network as outputs (Subasi, 2005).

$\left[\begin{array}{llll}0.9 & 0.1 & 0.1 & 0.1\end{array}\right]=$ Elongated bubble flow

$\left[\begin{array}{llll}0.1 & 0.9 & 0.1 & 0.1\end{array}\right]=$ Slug flow

$\left[\begin{array}{llll}0.1 & 0.1 & 0.9 & 0.1\end{array}\right]=$ Stratified flow

$\left[\begin{array}{llll}0.1 & 0.1 & 0.1 & 0.9\end{array}\right]=$ Stratified wavy flow

Table 3-1 Percentage of flow regimes in the experimental, training and testing data sets

\begin{tabular}{lcccccc}
\hline Flow Regime & Exp. Runs & $\%$ & Training Runs & $\%$ & Testing Runs & $\%$ \\
\hline Elongated bubble flow & 20 & 23.3 & 14 & 22.6 & 6 & 25 \\
Slug flow & 30 & 34.9 & 24 & 38.7 & 6 & 25 \\
Stratified flow & 20 & 23.3 & 14 & 22.6 & 6 & 25 \\
Stratified wavy flow & 16 & 18.6 & 10 & 16.1 & 6 & 25 \\
Total & 86 & 100 & 62 & 100 & 24 & 100 \\
\hline
\end{tabular}

The following back propagation algorithm training algorithms used in the network and their performance for the flow regime identification examined. The multilayer perceptron has three activation functions for regulating its output: pureline, logsig and tansig). In this study, the sigmoidal function was used throughout due to its properties such as ranges the output between 0 and 1, nonlinear paving the way for complex mappings of the input to the output and it is continuous and differentiable (Güler \& Übeyli 2006). Important aspects of the neural network development are architecture and the training process. Several training algorithms and neural network architectures have been test with different number of the hidden layer neurons of the network evaluated during the training process.

- Levenberg-Marquardt (LM)

- Scaled Conjugate Gradient(SCG)

- One Step Secant (OSS)

- Resilient Back-propagation(RP) 
- Quasi-Newton (BFGS)

- Bayesian Regulation (BR)

Before, the fusing of the neural network models, single levels neural models were tried out for designing the flow regime classifier but the results of the were not good enough. Subsequently, the single levels were integrated into the combined neural network. The neural networks with single layers were found to be superior to the two hidden layers in this experiment. The most efficient configuration for the network with PSD features was 12 neuron hidden layers while that of the DWT features was the 28 neuron in the hidden layers.

Combined neural network design: The combined network algorithm to determine flow regimes involves five sets of neural networks models: N1, N2, N3, N4 and N5. The models N1, N2, N3 and N4 are first level MLPNN formed with the input (features)-outputs relationships. The N5 model is the second level network of the combined network and it uses the outputs of the first level network as the inputs while using the same output as the first level network. Each member of the first level network produces four outputs corresponding to the flow regimes and these outputs are concatenated to form a vector for the input of the second level of the combined network. Hence, the combined neural network for the flow velocities training for the four different set of features extracted.

\section{Results and discussion}

In this study, horizontal gas-liquid two-phase four flow regimes were classified from ultrasonic Doppler signals processed using artificial neural network. The inputs to the neural network are features obtained from frequency bands of both power spectral density and discrete wavelet transforms. After the features extracted from the signals, they are normalised. Flow regimes classification models based on feed-forward multilayer perceptron neural network implemented in the MATLAB software package with neural network toolbox. In order improve the performance of the ANN classifier the networks were integrated into two-tier network called combined neural network (Übeyli \& Güler 2005). The total number of 85 datasets divided into training and testing sets and 62 data samples used for the training the network and 24 datasets were used for the testing the network.

\subsection{Feature extractions}

the features from the Doppler ultrasound signals of the flow extracted using two methods of features extractions were applied in this study. (1) Discrete wavelet transform was for generating the frequency bands by decomposing the Doppler signal and then applying each of these statistical measures: of mean, maximum, minimum and standard deviation to the results of the wavelet transform. (2) Frequency domain spectral analysis of the ultrasonic Doppler signal has been implemented using power spectral density based on the Fourier transform technique. The PSD spectrums is further averaged to be input or representative 
of the flow in the neural network, it is necessary to extract statistical moments from the spectra (Xie et al., 2004). Seven frequency bands or levels obtained from the details wavelet coefficients. So, for each of the dataset, we obtained twenty-eight features from the seven details wavelet coefficient levels and the statistical measure applied to the wavelet levels.

\subsection{Flow regime identification}

The testing of the flow regimes classifier were done using 24 datasets comprises six samples of the four flow regimes. After the flow regime classification neural network has learn the input-output relationship, then the two classifiers, one for each feature vectors, are tested with the datasets that the network has not seen before. The classification errors in the testing of new datasets are evaluated and shown in the form of confusion matrices in Figure 4-1 and Figure 4-2 for the PSD and DWT features respectively. The diagonal cells show the number of datasets that were correctly classified, and the off-diagonal cells show the misclassified datasets. The blue cell in the bottom right shows the total percent of correctly classified cases (in green) and the total percent of misclassified cases (in red). The result shows that the PSD trained network has missed three dataset in the classification, while the DWT trained network has misclassified only data point.

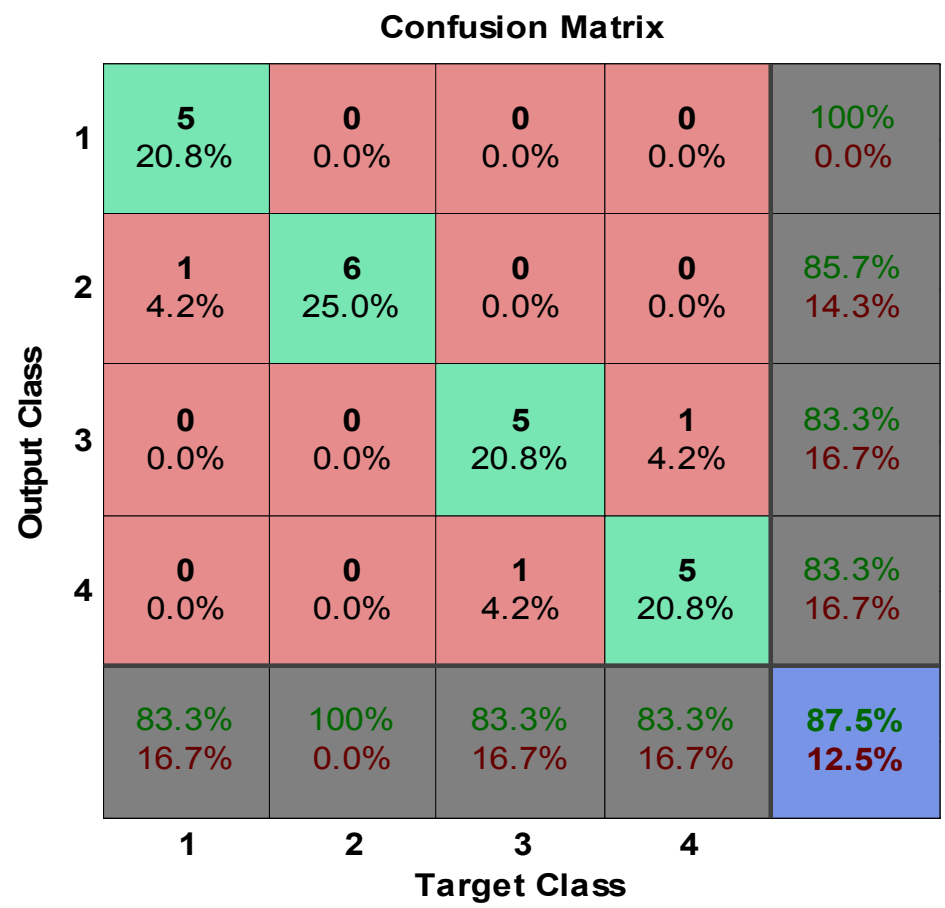

Figure 4-1 a confusion plot of the PSD features used in the combined neural network for flow regimes classification showing the classification errors that occurred 


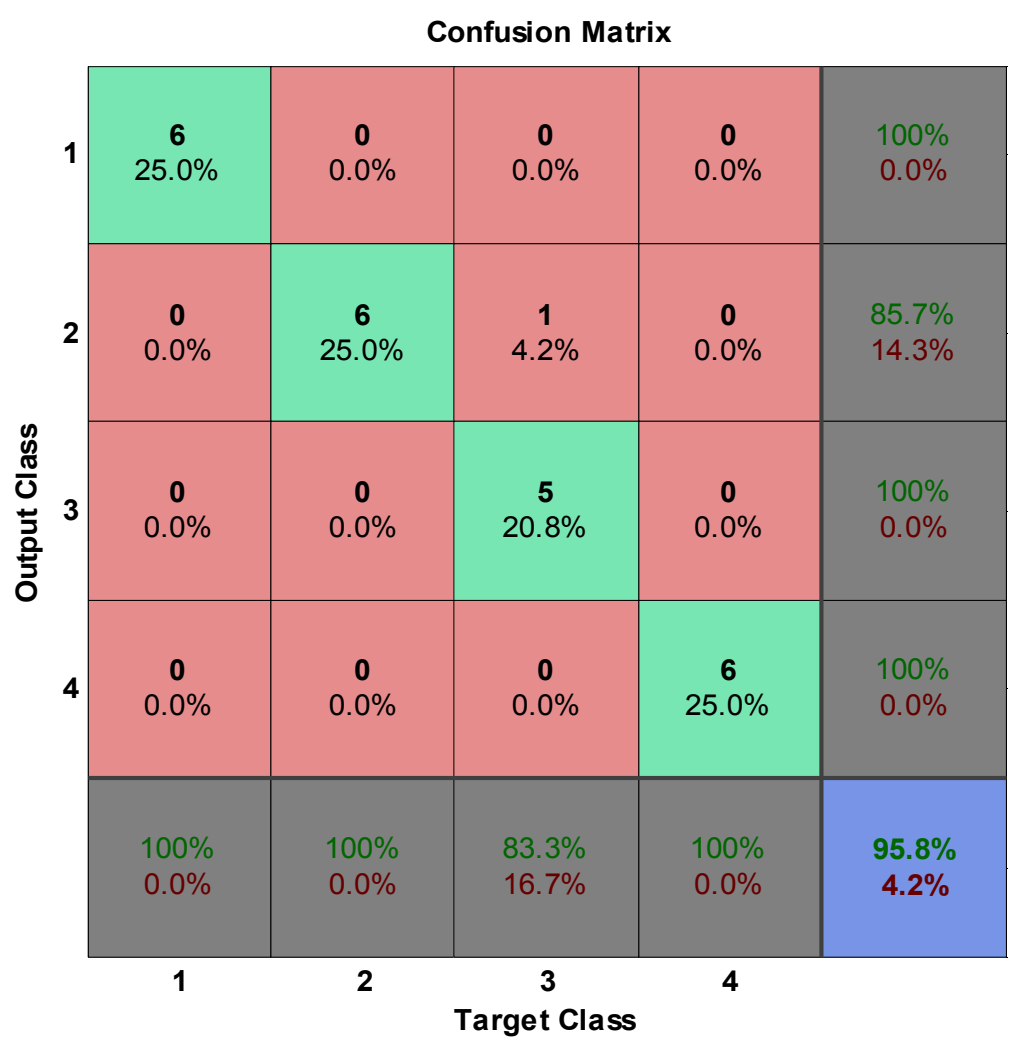

Figure 4-2 a confusion plot of the DWT features used in the combined neural network for flow regimes classification showing the classification errors that occurred

The flow regimes classification performance was evaluated in the form of confusion matrices. A misclassification is said to be occurred when the classifier fail to align a flow regime into the supposedly right group or class. From the Figure 4-1 and Figure 4-2, the summary of the classification accuracies of the each flow regimes and each method of classifier based on the features used in the development of the systems are presented in the Table 4-1. 
Table 4-1 classification accuracies for each of the flow regime and the total accuracy of each classifier

\begin{tabular}{|c|c|c|}
\hline Classifiers & $\begin{array}{l}\text { Flow regimes classification } \\
\text { accuracies }(\%)\end{array}$ & values \\
\hline Neural network with PSD features & $\begin{array}{l}\text { Elongated bubble flow } \\
\text { Slug flow } \\
\text { Stratified flow } \\
\text { Stratified wavy flow } \\
\text { Total classification accuracy }\end{array}$ & $\begin{array}{l}100 \\
85.7 \\
83.3 \\
83.3 \\
87.5\end{array}$ \\
\hline Neural network with DWT features & $\begin{array}{l}\text { Elongated bubble flow } \\
\text { Slug flow } \\
\text { Stratified flow } \\
\text { Stratified wavy flow } \\
\text { Total classification accuracy }\end{array}$ & $\begin{array}{l}100 \\
87.5 \\
100 \\
100 \\
95.8\end{array}$ \\
\hline
\end{tabular}

\subsubsection{Comparison of Visually Observed and Classified Flow Regimes}

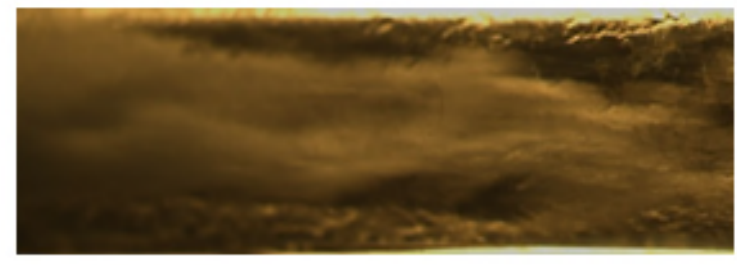

Slug flow

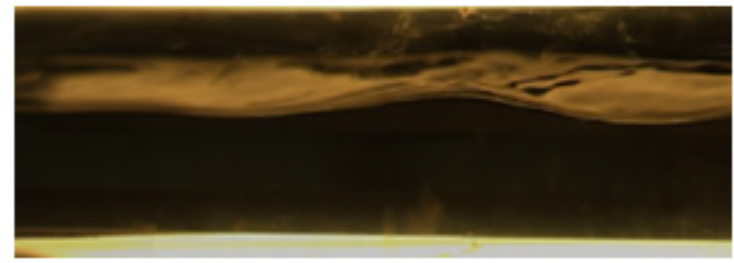

Stratified flow

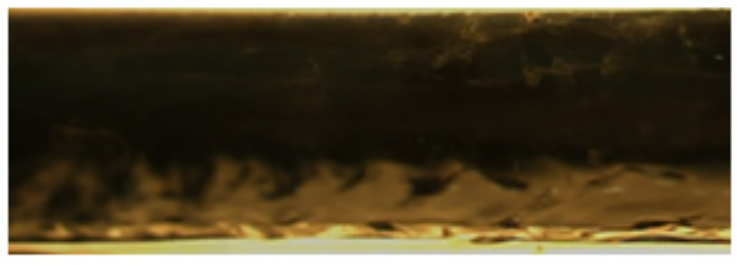

Stratified wavy flow

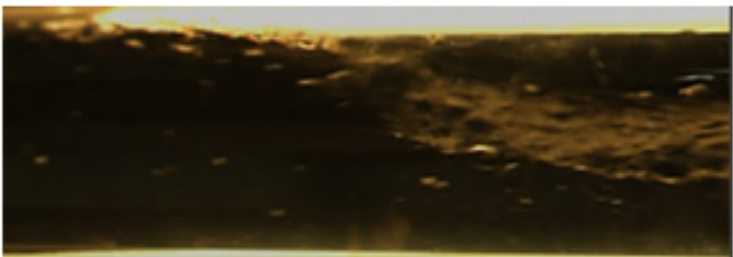

Bubbly flow

Figure 4-3 Typical flow regimes of the gas-liquid two-phase in a horizontal pipe recorded by a high speed camera 
Table 4-2 shows predicted the flow regimes with a classifier using the PSD features which is quite good with only three misclassifications out the 24 data records. The three misclassified flow regimes are cases no. 7 which identified from slug flow to elongated bubble flow, and no. 17 which is also identified from slug flow to elongated bubble flow. The last misclassified is number 23 which is from stratified-wavy flow to stratified flow. These misclassified flow patterns are denoted as $\mathrm{F}$ for false prediction whereas the successfully classified flow regimes denoted as $\mathrm{T}$ for true. Also, misclassified flow regimes are very similar to their actual targeted flow regimes. It has been found that only the nearby flow regimes were confused in the neural network as in the results of other work (Sun and Zhang, 2008).

Table 4-2 Classification performance of 7-16-4 MLPNN Levenberg-Marquardt trained with PSD features

\begin{tabular}{|c|c|c|c|c|}
\hline No. & $\begin{array}{l}\text { Superficial } \\
\text { gas velocity } \\
\mathrm{m} / \mathrm{s}\end{array}$ & $\begin{array}{l}\text { Superficial } \\
\text { water velocity } \\
\mathrm{m} / \mathrm{s}\end{array}$ & Observed flow regime & $\begin{array}{l}\text { Classified flow } \\
\text { regimes with } \\
\text { MLPNN }\end{array}$ \\
\hline 1. & 0.5 & 0.4 & Elongated bubble flow & $\mathrm{T}$ \\
\hline 2. & 0.7 & 0.4 & Elongated bubble flow & $\mathrm{T}$ \\
\hline 3. & 0.9 & 0.4 & Elongated bubble flow & $\mathrm{T}$ \\
\hline 4. & 1.0 & 0.4 & Elongated bubble flow & $\mathrm{T}$ \\
\hline 5. & 1.3 & 0.4 & Elongated bubble flow & $\mathrm{T}$ \\
\hline 6. & 1.5 & 0.4 & Elongated bubble flow & $\mathrm{T}$ \\
\hline 7. & 0.5 & 0.7 & Elongated bubble flow & $\mathrm{F}$ \\
\hline 8. & 0.7 & 1.0 & Slug flow & $\mathrm{T}$ \\
\hline 9. & 0.9 & 0.7 & Slug flow & $\mathrm{T}$ \\
\hline 10. & 1.1 & 0.5 & Slug flow & $\mathrm{T}$ \\
\hline 11. & 1.3 & 0.7 & Slug flow & $\mathrm{T}$ \\
\hline 12. & 1.5 & 1.0 & Slug flow & $\mathrm{T}$ \\
\hline 13. & 0.5 & 0.1 & Stratified flow & $\mathrm{T}$ \\
\hline 14. & 0.5 & 0.1 & Stratified flow & $\mathrm{T}$ \\
\hline 15. & 0.5 & 0.1 & Stratified flow & $\mathrm{T}$ \\
\hline 16. & 0.7 & 0.1 & Stratified flow & $\mathrm{T}$ \\
\hline 17. & 0.7 & 0.1 & Stratified flow & $F$ \\
\hline 18. & 0.7 & 0.1 & Stratified flow & $\mathrm{T}$ \\
\hline 19. & 0.1 & 0.9 & Stratified wavy flow & $\mathrm{T}$ \\
\hline 20. & 0.1 & 0.9 & Stratified wavy flow & $\mathrm{T}$ \\
\hline
\end{tabular}




\begin{tabular}{lllll}
\hline 21. & 0.1 & 1.1 & Stratified wavy flow & $\mathrm{T}$ \\
22. & 0.1 & 1.1 & Stratified wavy flow & $\mathrm{T}$ \\
23. & 0.1 & 1.1 & Stratified wavy flow & $\mathrm{F}$ \\
24. & 0.1 & 1.3 & Stratified wavy flow & $\mathrm{T}$ \\
\hline
\end{tabular}

Similarly, in the Table 4-3, the DWT features from the test dataset is applied to the trained neural network. This classifier was able to match all flow regimes except only one in the case number no. 18 where it was misclassified from stratified flow to slug flow. Importantly, its overall performance is that it can classify the flow pattern up to $96 \%$. As a result, the combined neural network built using the MLPNN and DWT features has higher quality of classification than the one trained with PSD features. These results are similar to flow pattern classifications works found in previous studies (Hernandez et al., 2006; Sun and Zhang, 2008).

Table 4-3 Classification performance of the 28-10-4 Levenberg-Marquardt selected for DWT features

\begin{tabular}{|c|c|c|c|c|}
\hline No. & $\begin{array}{l}\text { Superficial } \\
\text { gas velocity } \\
\mathrm{m} / \mathrm{s}\end{array}$ & $\begin{array}{l}\text { Superficial } \\
\text { water } \\
\text { velocity } \mathrm{m} / \mathrm{s}\end{array}$ & Observed flow regimes & $\begin{array}{l}\text { Classified } \\
\text { regimes } \\
\text { MLPNN }\end{array}$ \\
\hline 1. & 0.5 & 0.4 & Elongated bubble flow & $\mathrm{T}$ \\
\hline 2. & 0.7 & 0.4 & Elongated bubble flow & $\mathrm{T}$ \\
\hline 3. & 0.9 & 0.4 & Elongated bubble flow & $\mathrm{T}$ \\
\hline 4. & 1.0 & 0.4 & Elongated bubble flow & $\mathrm{T}$ \\
\hline 5. & 1.3 & 0.4 & Elongated bubble flow & $\mathrm{T}$ \\
\hline 6. & 1.5 & 0.4 & Elongated bubble flow & $\mathrm{T}$ \\
\hline 7. & 0.5 & 0.7 & Slug flow & $\mathrm{T}$ \\
\hline 8. & 0.7 & 1.0 & Slug flow & $\mathrm{T}$ \\
\hline 9. & 0.9 & 0.7 & Slug flow & $\mathrm{T}$ \\
\hline 10. & 1.1 & 0.5 & Slug flow & $\mathrm{T}$ \\
\hline 11. & 1.3 & 0.7 & Slug flow & $\mathrm{T}$ \\
\hline 12. & 1.5 & 1.0 & Slug flow & $\mathrm{T}$ \\
\hline 13. & 0.5 & 0.1 & Stratified flow & $\mathrm{T}$ \\
\hline 14. & 0.5 & 0.1 & Stratified flow & $\mathrm{T}$ \\
\hline 15. & 0.5 & 0.1 & Stratified flow & $\mathrm{T}$ \\
\hline 16. & 0.7 & 0.1 & Stratified flow & $\mathrm{T}$ \\
\hline 17. & 0.7 & 0.1 & Stratified flow & $\mathrm{T}$ \\
\hline 18. & 0.7 & 0.1 & Stratified flow & $F$ \\
\hline
\end{tabular}




\begin{tabular}{lllll} 
19. & 0.1 & 0.9 & Stratified wavy flow & $\mathrm{T}$ \\
20. & 0.1 & 0.9 & Stratified wavy flow & $\mathrm{T}$ \\
21. & 0.1 & 1.1 & Stratified wavy flow & $\mathrm{T}$ \\
22. & 0.1 & 1.1 & Stratified wavy flow & $\mathrm{T}$ \\
23. & 0.1 & 1.1 & Stratified wavy flow & $\mathrm{T}$ \\
24. & 0.1 & 1.3 & Stratified wavy flow & $\mathrm{T}$ \\
\hline
\end{tabular}

\subsubsection{Comparison of the Performance of PSD and DWT Features}

Performances of the six MLPNN structures trained using six different training algorithms for the flow regimes classification compared. The comparison illustrated in the Figure 4-4. the training algorithm with a single hidden layer gives the best performing network.

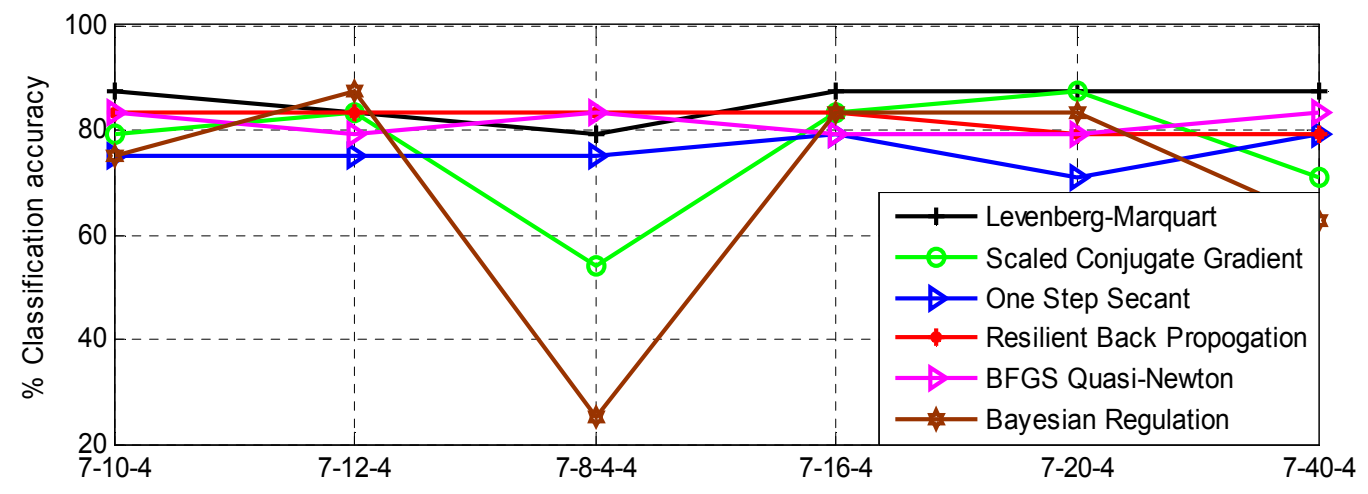

(a) MLPNN structure with PSD features

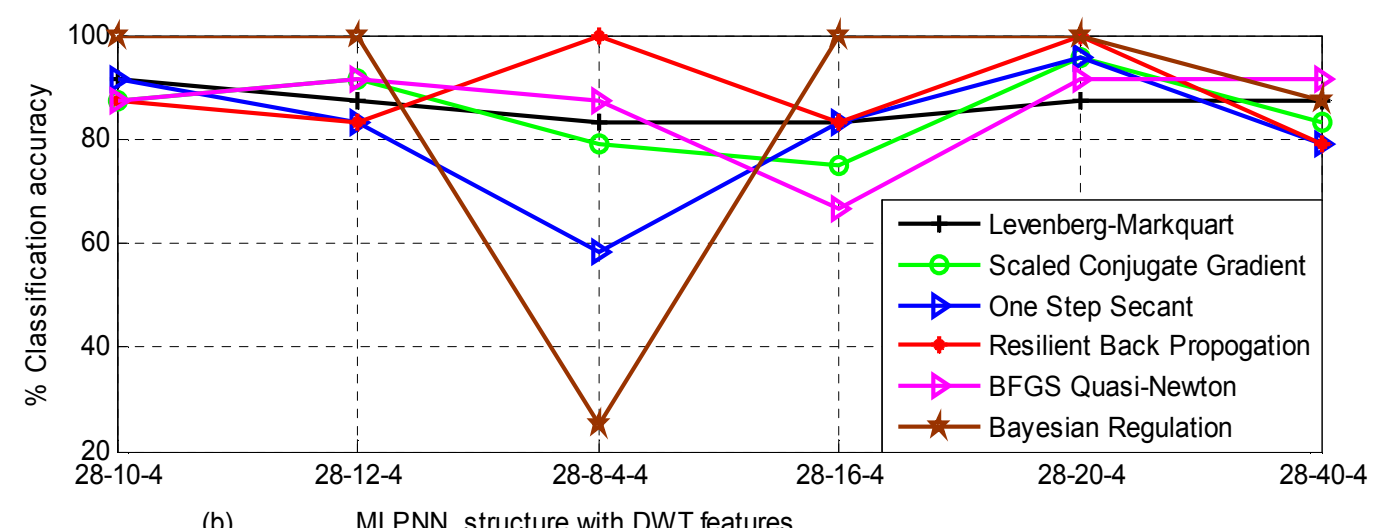

Figure 4-4 performance of various MLPNN structures and the training algorithms for both (a) Using features trained with PSD extraction (b) Using DWT extracted features.

\section{Conclusion}

In this paper development of a clamp-on continuous wave Doppler ultrasound sensor and artificial neural network (ANN) for gas-liquid two-phase flow regimes classification was performed. The ultrasound signals are processed using the methods of power spectral density and discrete wavelet transforms for extraction of input features to the ANN models. 
Multilayer perceptron neural network developed into a combined neural network of twolevels as the classifier and the two methods of feature extractions produced for the network. Comparisons of the performances of the two classifier models assessed. Four two-phase flow regimes such as slug flow, stratified flow, elongated bubble flow and stratified wavy have been classified using the method developed.

The results show that ultrasound signal features of the two phase flow obtained using the discrete wavelet transform performs better accurate classification than compared to the features extracted with power spectral density methods. The combined neural network models developed for the classification using the PSD features and for the DWT features have accuracies of $87 \%$ and $95.6 \%$ respectively. In conclusion, the present study has demonstrated that discrete wavelet transforms feature extraction and the MLPNN classifier has met the industrial requirement of flow regime classification(Sun et al. 2013).

In contrast to the invasive instruments used in other works, this approach is very important for industrial application given that the sensor used is non-invasive, non-radioactive and it is ultrasound technology. Based on the analysis of the experimental results, the proposed method is able provide objective classification of four flow regimes in the horizontal pipe. Other ultrasonic methods reported in the literature employed pulse echo ultrasound plus neural network or subjective methods of flow regimes indemnification (Jha et al. 2013; Figueiredo et al. 2016). The key strengths of the neural network based methods are fast classification and flexible procedure for finding good nonlinear solutions.

The continuous wave Doppler sensor is suitable for monitoring flow processes that are ultrasonically reflection fluids such as crude oil, petrocarbons, oil-gas and oil-water mixtures. Importantly, the sensor can fits well on existing pipework and it is suitable for both plastic and metallic pipes. However, on horizontal pipe, it is important to mount the sensor at the bottom of the pipe to avoid gas voids in the upper section of the pipe. Poor coupling of the sensor with the pipe, gas voids or bends would temper with strength of the signal received. The results of the present study demonstrated that the proposed approach of wavelet transforms and MLPNN classifier has met the industrial requirement of flow regime classification(Sun et al. 2013).

Further studies are needed in the application of this clamp-on objective flow regime classification system to investigate oil-water two-phase flow. Especially for deployment of this technology to address requirement of clamp-on ultrasound flow monitoring meter for oil well testing. Also, more studies are recommended on the feasibility of information of the ultrasound Doppler sensor and void fraction measurement such as gamma densitometer which would make the system a complete multiphase flow meter. 


\section{Acknowledgement}

the authors gratefully acknowledge the financial support from the Petroleum Technology Development Fund (Nigeria) under the Overseas Scholarship Scheme. 


\section{References}

Arubi, I.M.T., 2011. Multiphase flow measurement using gamma-based techniques. Cranfield University. Available at: http://dspace.lib.cranfield.ac.uk/handle/1826/8347.

Arvoh, B.K., Hoffmann, R. \& Halstensen, M., 2012. Estimation of volume fractions and flow regime identification in multiphase flow based on gamma measurements and multivariate calibration. Flow Measurement and Instrumentation, 23(1), pp.56-65.

Banerjee, S. \& Lahey Jr, R.T., 1981. Advances in two-phase flow instrumentation. In Advances in Nuclear Science and Technology. Springer, pp. 227-414.

Basheer, I.A. \& Hajmeer, M., 2000. Artificial neural networks: fundamentals , computing , design , and application. Journal of Microbiological Methods, 43, pp.3-31. Available at: http://www.sciencedirect.com/science/article/pii/S0167701200002013.

Beale, M.H., Hagan, M.T. \& Demuth, H.B., 2013. MATLAB Neural Network Toolbox User's Guide 2013b, Natick, Massachusetts, United States.: The MathWorks, Inc.,.

Bendjama, H. et al., 2015. Selection of Wavelet Decomposition Levels for Vibration Monitoring of Rotating Machinery. In The Ninth International Conference on Advanced Engineering Computing and Applications in Sciences ADVCOMP 2015. Nice, France: IARIA XPS Press, pp. 96-100.

Bishop, C.M. \& James, G.D., 1993. Analysis of multiphase flows using dual-energy gamma densitometry and neural networks. Nuclear Instruments and Methods in Physics Research Section A: Accelerators, Spectrometers, Detectors and Associated Equipment, 327(2), pp.580-593.

Blaney, S. \& Yeung, H., 2008. Investigation of the exploitation of a fast-sampling single gamma densitometer and pattern recognition to resolve the superficial phase velocities and liquid phase water cut of vertically upward multiphase flows. Flow Measurement and Instrumentation, 19(2), pp.57-66. Available at: http://www.sciencedirect.com/science/article/pii/S095559860700074X.

Cai, S. et al., 1994. Neural network based objective flow regime identification in air-water two phase flow. The Canadian Journal of Chemical Engineering, 72(3), pp.440-445.

Canière, H. et al., 2007. Horizontal two-phase flow characterization for small diameter tubes with a capacitance sensor. Measurement Science and Technology, 18(9), pp.28982906.

Chakraborty, S. et al., 2009. Void fraction measurement in two-phase flow processes via symbolic dynamic filtering of ultrasonic signals. Measurement Science and Technology, 20(2), p.23001. 
Chang, J.S. \& Morala, E.C., 1990. Determination of two-phase interfacial areas by an ultrasonic technique. Nuclear Engineering and Design, 122(1-3), pp.143-156. Available at: http://www.sciencedirect.com/science/article/pii/002954939090203A.

Corneliussen, S. et al., 2005. Handbook of multiphase flow metering. Norwegian Society for Oil and Gas Measurement (NFOGM), Revision, 2.

Drahoš, J. \& Čermák, J., 1989. Diagnostics of gas-liquid flow patterns in chemical engineering systems. Chemical Engineering and Processing: Process ..., 26, pp.147164. Available at: http://www.sciencedirect.com/science/article/pii/025527018990007X.

Evans, D.H. \& McDicken, W.N., 2000. Doppler ultrasound: physics, instrumentation, and signal processing, Wiley Chichester.

Falcone, G., Hewitt, G.F. \& Alimonti, C., 2009. Multiphase Flow Metering: Principles and Applications, Elsevier Science.

Fan, S. et al., 2013. Velocity characteristics of slug body and film for two-phase gas-liquid slug flow using ultrasonic techniques. 8th International Conference on Multiphase Flow ICMF 2013, Jeju, Korea, May 26 - 31, 2013, pp.1-5.

Fan, S. \& Yan, T., 2014. Two-phase air-water slug flow measurement in horizontal pipe using conductance probes and neural network. IEEE Transactions on Instrumentation and Measurement, 63(2), pp.456-466.

Figueiredo, M.M.F. et al., 2016. The use of an ultrasonic technique and neural networks for identification of the flow pattern and measurement of the gas volume fraction in multiphase flows. Experimental Thermal and Fluid Science, 70, pp.29-50. Available at: http://dx.doi.org/10.1016/j.expthermflusci.2015.08.010.

Filletti, E.R. \& Seleghim jr, Paulo, 2010. Nonintrusive measurement of interfacial area and volumetric fraction in dispersed two-phase flows using a neural network to process acoustic signals-A numerical investigation. International Journal for Numerical Methods in Biomedical Engineering, 28(1), pp.72-86. Available at: http://onlinelibrary.wiley.com/doi/10.1002/cnm.1494/full.

Güler, İ. \& Übeyli, E.D., 2006. A recurrent neural network classifier for Doppler ultrasound blood flow signals. Pattern Recognition Letters, 27(13), pp.1560-1571.

Hernández, L. et al., 2006. Fast classification of two-phase flow regimes based on conductivity signals and artificial neural networks. Measurement Science and Technology, 17(6), pp.1511-1521.

Hu, H.L. et al., 2011. Identification of Gas-Solid Two-Phase Flow Regimes Using HilbertHuang Transform and Neural-Network Techniques. Instrumentation Science \& 
Technology, 39(2), pp.198-210. Available at: http://www.tandfonline.com/doi/abs/10.1080/10739149.2010.545852.

Jain, a. K., Duin, R.P.W. \& Mao, J., 2000. Statistical pattern recognition: a review. IEEE Transactions on Pattern Analysis and Machine Intelligence, 22(1), pp.4-37. Available at:

http://ieeexplore.ieee.org/ielx5/34/17859/00824819.pdf?tp=\&arnumber=824819\&isnum ber=17859lnhttp://ieeexplore.ieee.org/xpls/abs_all.jsp?arnumber=824819.

Jha, D.K. et al., 2013. Classification of Two-Phase Flow Patterns by Ultrasonic Sensing. Journal of Dynamic Systems, Measurement, and Control, 135(2), p.24503.

Kandaswamy, A. et al., 2004. Neural classification of lung sounds using wavelet coefficients. Computers in biology and medicine, 34(6), pp.523-537. Available at: http://www.sciencedirect.com/science/article/pii/S0010482503000921.

Kouame, D. et al., 2003. High resolution processing techniques for ultrasound Doppler velocimetry in the presence of colored noise. II. Multiplephase pipe-flow velocity measurement. Ultrasonics, Ferroelectrics and Frequency Control, IEEE Transactions on, 50(3), pp.267-278.

Kv, S. \& Roy, B.K., 2012. An Intelligent Flow Measurement Technique using Ultrasonic Flow Meter with Optimized Neural Network. International Journal of Control and Automation, 5(4), pp.185-196.

Liu, R.P. et al., 2001. A neural network to correct mass flow errors caused by two-phase flow in a digital coriolis mass flowmeter. Flow Measurement and Instrumentation, 12(1), pp.53-63. Available at: http://www.sciencedirect.com/science/article/pii/S0955598600000455.

Luntta, E. \& Halttunen, J., 1999. Neural network approach to ultrasonic flow measurements. Flow Measurement and Instrumentation, 10(1), pp.35-43.

Matsumoto, S. \& Suzuki, M., 1984. Statistical analysis of fluctuations of froth pressure on perforated plates without downcomers. International Journal of Multiphase Flow, 10(2), pp.217-228.

Meribout, M. et al., 2010. A Multisensor intelligent device for real-time multiphase flow metering in oil fields. Instrumentation and Measurement, IEEE Transactions on, 59(6), pp.1507-1519.

Mi, Y., Ishii, M. \& Tsoukalas, L.H., 2001. Flow regime identification methodology with neural networks and two-phase flow models. Nuclear Engineering and Design, 204(1), pp.87100. 
Misiti, M. et al., 1997. Wavelet toolbox. For use with MATLAB., Natick, MA: The MathWorks Inc.

Murai, Y. et al., 2010. Ultrasonic detection of moving interfaces in gas-liquid two-phase flow. Flow Measurement and Instrumentation, 21(3), pp.356-366.

Ozbayoglu, E.M. \& Ozbayoglu, M. a., 2009. Estimating Flow Patterns and Frictional Pressure Losses of Two-Phase Fluids in Horizontal Wellbores Using Artificial Neural Networks. Petroleum Science and Technology, 27(2), pp.135-149.

Rajan, V.S. V, Ridley, R.K. \& Rafa, K.G., 1993. Multiphase flow measurement techniques--A review. Journal of Energy Resources Technology;(United States), 115(3), pp.151-161. Available at: http://energyresources.asmedigitalcollection. asme.org/article. aspx?articleid=1413270.

Rosa, E.S. et al., 2010. Performance comparison of artificial neural networks and expert systems applied to flow pattern identification in vertical ascendant gas-liquid flows. International Journal of Multiphase Flow, 36(9), pp.738-754. Available at: http://dx.doi.org/10.1016/j.jpmultiphaseflow.2010.05.001.

Sanderson, M.L. \& Yeung, H., 2002. Guidelines for the use of ultrasonic non-invasive metering techniques. Flow Measurement and Instrumentation, 13(4), pp.125-142.

Santoso, B. et al., 2012. The Identification of Gas-liquid Co-current Two Phase Flow Pattern in a Horizontal Pipe Using the Power Spectral Density and the Artificial Neural Network (ANN). Modern Applied Science, 6(9), pp.56-67. Available at: http://www.ccsenet.org/journal/index.php/mas/article/view/20121.

Shaban, H. \& Tavoularis, S., 2014. Measurement of gas and liquid flow rates in two-phase pipe flows by the application of machine learning techniques to differential pressure signals. International Journal of Multiphase Flow, 67, pp.106-117. Available at: http://www.sciencedirect.com/science/article/pii/S0301932214001608.

Shang, Z. et al., 2004. An investigation of two-phase flow instability using wavelet signal extraction technique. Nuclear Engineering and Design, 232(2), pp.157-163.

Subasi, A., 2005. Automatic recognition of alertness level from EEG by using neural network and wavelet coefficients. Expert Systems with Applications, 28(4), pp.701-711.

Sun, Z. \& Zhang, H., 2008. Neural networks approach for prediction of gas-liquid two-phase flow pattern based on frequency domain analysis of vortex flowmeter signals. Measurement Science and Technology, 19(1), p.15401.

Sun, Z.Q., Shao, S. \& Gong, H., 2013. Gas-liquid Flow Pattern Recognition Based on Wavelet Packet Energy Entropy of Vortex-induced Pressure Fluctuation. Measurement 
Science Review, 13(2), pp.83-88. Available at: <Go to ISI>://WOS:000318150800006.

Sunde, C., Avdic, S. \& Pázsit, I., 2005. Classification of two-phase flow regimes via image analysis and a neuro-wavelet approach. Progress in Nuclear Energy, 46(3-4), pp.348358. Available at: http://linkinghub.elsevier.com/retrieve/pii/S0149197005000302.

Übeyli, E.D. \& Güler, İ., 2005. Improving medical diagnostic accuracy of ultrasound Doppler signals by combining neural network models. Computers in biology and medicine, 35(6), pp.533-554.

Wada, S., Kikura, H. \& Aritomi, M., 2006. Pattern recognition and signal processing of ultrasonic echo signal on two-phase flow. Flow Measurement and Instrumentation, 17(4), pp.207-224. Available at: http://www.sciencedirect.com/science/article/pii/S0955598605001081.

Wang, Z. et al., 2013. A comparative study of delay time identification by vibration energy analysis in millisecond blasting. International Journal of Rock Mechanics and Mining Sciences, 60, pp.389-400. Available at: http://www.scopus.com/inward/record.url?eid=2-s2.084874563248\&partnerID=40\&md5=10f923405bf6ac2f8131d71e8ffdc48e.

Wolpert, D.H., 1992. Stacked generalization. Neural Networks, 5(2), pp.241-259.

Xie, T., Ghiaasiaan, S.M. \& Karrila, S., 2004. Artificial neural network approach for flow regime classification in gas-liquid-fiber flows based on frequency domain analysis of pressure signals. Chemical Engineering Science, 59(11), pp.2241-2251. Available at: http://www.sciencedirect.com/science/article/pii/S0009250904001381.

Xie, T., Ghiaasiaan, S.M. \& Karrila, S., 2003. Flow Regime Identification in Gas / Liquid / Pulp Fiber Slurry Flows Based on Pressure Fluctuations Using Artificial Neural Networks. Industrial and Engineering Chemistry Research, 42, pp.7017-7024. 